\title{
Localized waves and interaction solutions to the fractional generalized CBS-BK equation arising in fluid mechanics
}

\author{
Jalil Manafian 1* (D, Onur Alp Ilhan², Ladan Avazpour ${ }^{3}$ and As'ad Alizadeh ${ }^{4}$
}

\section{"Correspondence:}

j_manafianheris@tabrizu.ac.ir

'Department of Applied

Mathematics, Faculty of

Mathematical Sciences, University

of Tabriz, Tabriz, Iran

Full list of author information is

available at the end of the article

\section{Springer}

\begin{abstract}
The Hirota bilinear method is employed for searching the localized waves, lump-solitons, and solutions between lumps and rogue waves for the fractional generalized Calogero-Bogoyavlensky-Schiff-Bogoyavlensky-Konopelchenko (CBS-BK) equation. We probe three cases including lump (combination of two positive functions as polynomial), lump-kink (combination of two positive functions as polynomial and exponential function) called the interaction between a lump and one line soliton, and lump-soliton (combination of two positive functions as polynomial and hyperbolic cos function) called the interaction between a lump and two-line solitons. At the critical point, the second-order derivative and the Hessian matrix for only one point will be investigated and the lump solution has one maximum value. The moving path of the lump solution and also the moving velocity and the maximum amplitude will be obtained. The graphs for various fractional orders $\alpha$ are plotted to obtain 3D plot, contour plot, density plot, and 2D plot. The physical phenomena of this obtained lump and its interaction soliton solutions are analyzed and presented in figures by selecting the suitable values. That will be extensively used to report many attractive physical phenomena in the fields of fluid dynamics, classical mechanics, physics, and so on.
\end{abstract}

Keywords: Hirota bilinear method; Lump-solitons; Fractional generalized Calogero-Bogoyavlensky-Schiff-Bogoyavlensky-Konopelchenko equation; Hessian matrix

\section{Introduction}

The nonlinear partial differential equation is a physical and natural model which can be used for model constructs by scientists and researchers. Different types of differential equations of both ODEs and PDEs in various fields of science, like fluid flow, mechanics, and biology, are expressed in the special forms $[1,2]$. There is no particular method for accessing the exact type solutions of nonlinear PDEs but some approximate and analytical solutions have been determined [3, 4]. As is well known, the model of many natural phenomena and the differential equations in the sciences and engineering are nonlinear and it is very important to obtain analytically or numerically accurate solutions. In order to achieve this goal, various methods have been developed for linear and nonlin-

(c) The Author(s) 2021. This article is licensed under a Creative Commons Attribution 4.0 International License, which permits use, sharing, adaptation, distribution and reproduction in any medium or format, as long as you give appropriate credit to the original author(s) and the source, provide a link to the Creative Commons licence, and indicate if changes were made. The images or other third party material in this article are included in the article's Creative Commons licence, unless indicated otherwise in a credit line to the material. If material is not included in the article's Creative Commons licence and your intended use is not permitted by statutory regulation or exceeds the permitted use, you will need to obtain permission directly from the copyright holder. To view a copy of this licence, visit http://creativecommons.org/licenses/by/4.0/. 
ear equations, such as the Exp-function method [5], the homotopy analysis method [6], the homotopy perturbation method [7], the (G'/G)-expansion method [8], the improved $\tan (\phi / 2)$-expansion method [9, 10], the Hirota bilinear method [11, 12, 59-67], the $\mathrm{He}$ variational principle [13, 14], the binary Darboux transformation [15], the Lie group analysis [16, 17], the Bäcklund transformation method [18], and the multiple Exp-function method $[19,20]$. Moreover, many powerful methods have been used to investigate the new properties of mathematical models which are symbolizing serious real world problems $[21,22,68,69]$. The utilized methods which employed by powerful researchers are such methods as the Exp-function method, the multiple Exp-function method, (G'-G)expansion method-but we should not forget to mention that these methods continue to attract a wave of criticism. This criticism is focused on two aspects of these methods. First of all, it has been demonstrated that the mentioned methods can produce wrong solutions. Secondly, these methods cannot produce necessary and sufficient conditions for the existence of analytic solutions, neither in the system parameter space nor in the space of initial conditions. These aspects can be useful for investigating in future research and combining these methods in work which will have a link with special transformations.

The main idea of the following equations is finding the exact solution of any models which can be expressed by a Hirota bilinear method. Big varieties of mathematical and physical phenomena are governed by NLPDEs which play a crucial role in the nonlinear sciences. It provides much physical information and more insight into the physical aspects of the problem and thus leads to further applications. Bogoyavlensky introduced a model equation describing the nonisospectral scattering problems [23], namely, the $(2+1)$-dimensional Bogoyavlenski equation

$$
\begin{aligned}
& 4 \Psi_{t}+\Psi_{x x y}-4 \Psi^{2} \Psi_{y}-4 \Psi_{x} \Phi=0, \\
& \Psi \Psi_{y}=\Phi_{x} .
\end{aligned}
$$

Kudryashov and Pickering [24] proposed the above equation as a member of a $(2+1)$ Schwarzian breaking soliton hierarchy. Clarkson and co-authors [25] investigated Eq. (1.1) as one of the equations associated to nonisospectral scattering problems. Estevez and Prada [26] presented a generalization of the sine-Gordon equation that possesses the Painlevé feature. Zhran and Khater [27] probed the Bogoyavlensky equation by utilizing the modified extended tanh-function method. The authors of [28] showed that the above equation is a modified version of the following nonlinear equation:

$$
4 \Psi_{x t}+8 \Psi_{x} \Psi_{x y}+4 \Psi_{y} \Psi_{x x}+\Psi_{x x x y}=0,
$$

which is called the breaking soliton equation. Also, Eq. (1.2) is a particular version of the Bogoyavlensky-Konopelchenko (BK) equation given as

$$
a \Psi_{x t}+b \Psi_{x x x x}+c \Psi_{x x y}+d \Psi_{x} \Psi_{x x}+e \Psi_{x} \Psi_{x y}+k \Psi_{x x} \Psi_{y}=0 .
$$

Equation BK explains the $(2+1)$-dimensional interaction of a Riemann wave propagation along the $y$-axis with a long wave along the $x$-axis, and it also is a two-dimensional generalization of the well-known Korteweg-de Vries equation [29, 30]. This study is aimed 
at investigating the following generalized Bogoyavlensky-Konopelchenko (BK) equation [31]:

$$
\Psi_{t}+\alpha\left(6 \Psi \Psi_{x}+\Psi_{x x x}\right)+\beta\left(\Psi_{x x y}+3 \Psi \Psi_{y}+3 \Psi_{x} \Phi_{y}\right)+\gamma_{1} \Psi_{x}+\gamma_{2} \Psi_{y}+\gamma_{3} \Phi_{y y}=0,
$$

in which $\Phi_{x}=\Psi$, and $\alpha, \beta, \gamma_{1}, \gamma_{2}$, and $\gamma_{3}$ are determined values. Equation (1.4) can be written as

$$
\begin{aligned}
& \Phi_{x t}+\alpha\left(6 \Phi_{x} \Phi_{x x}+\Phi_{x x x x}\right)+\beta\left(\Phi_{x x x y}+3 \Phi_{x} \Phi_{x y}+3 \Phi_{x x} \Phi_{x y}\right) \\
& +\gamma_{1} \Phi_{x x}+\gamma_{2} \Phi_{x y}+\gamma_{3} \Phi_{y y}=0
\end{aligned}
$$

and by applying the bilinear transformation $\Psi=2(\ln f)_{x x}$ and $\Phi=2(\ln f)_{x}$ Eq. (1.5) is transformed to the bilinear form

$$
\begin{aligned}
& \left(\alpha D_{x}^{4}+\beta D_{x}^{3} D_{y}+D_{t} D_{x}+\gamma_{1} D_{x}^{2}+\gamma_{2} D_{x} D_{y}+\gamma_{3} D_{y}^{2}\right) f . f=0, \\
& D_{x}^{4} f . f=2\left(\left(f_{x x x x}-4 f_{x} f_{x x x}+3 f_{x x}^{2}\right), \quad D_{x}^{3} D_{y} f . f=2\left(f_{x x x y}-f_{y} f_{x x x}-3 f_{x} f_{x x y}+3 f_{x x} f_{x y}\right),\right. \\
& D_{x}^{2} f . f=2\left(f_{x x}-f_{x}^{2}\right), \quad D_{x} D_{t} f \cdot f=2\left(f_{x t}-f_{x} f_{t}\right), D_{x} D_{y} f . f=2\left(f_{x y}-f_{x} f_{y}\right), \\
& D_{y}^{2} f . f=2\left(f_{y y}-f_{y}^{2}\right) .
\end{aligned}
$$

The aim of this study is to construct the invariant solutions of the $(2+1)$-dimensional fractional generalized CBS-BK equation in the following form:

$$
\begin{aligned}
& D_{t}^{\alpha} \Psi+\Psi_{x x y}+3 \Psi_{x} \Psi_{y}+\delta_{1} \Psi_{y}+\delta_{2} \Phi_{y y}+\delta_{3} \Psi_{x} \\
& \quad+\delta_{4}\left(3 \Psi_{x}^{2}+\Psi_{x x x}\right)+\delta_{5}\left(3 \Phi_{y y}^{2}+\Psi_{y y y y}\right) \\
& \quad+\delta_{6}\left(3 \Psi_{y} \Phi_{y y}+\Psi_{y y y}\right)=0, \quad \Psi_{x}=\Phi, 0<\alpha \leq 1,
\end{aligned}
$$

in which $\delta_{i}, i=1, \ldots, 6$, are the determined values. By employing the following fractional transformation [32]:

$$
\tau=\frac{t^{\alpha}}{\Gamma(\alpha+1)}
$$

Equation (1.7) changes to the nonlinear fractional generalized CBS-BK equation as follows:

$$
\begin{aligned}
& \Psi_{\tau}+\Psi_{x x y}+3 \Psi_{x} \Psi_{y}+\delta_{1} \Psi_{y}+\delta_{2} \Phi_{y y}+\delta_{3} \Psi_{x}+\delta_{4}\left(3 \Psi_{x}^{2}+\Psi_{x x x}\right)+\delta_{5}\left(3 \Phi_{y y}^{2}+\Psi_{y y y y}\right) \\
& \quad+\delta_{6}\left(3 \Psi_{y} \Phi_{y y}+\Psi_{y y y}\right)=0, \quad \Psi_{x}=\Phi, 0<\alpha \leq 1 .
\end{aligned}
$$

The propagation and the dynamical behavior of these solutions can be analyzed for the different choices of $\alpha$, the fractional order. When $\alpha=1, N=1$ in an $N$-soliton, it is verified that the velocity of the soliton cannot be influenced by the variable coefficients. Furthermore, the shape and the amplitude of the soliton cannot be affected. For $\alpha=1$, when the arbitrary constants are supposed $\delta_{3}=\delta_{4}=\delta_{5}=\delta_{6}=0$, Eq. (1.9) changes as a generalized Calogero-Bogoyavlensky-Schiff (CBS) equation where has been cited in Refs. [33, 34]. 
While the arbitrary constants supposed $\delta_{5}=\delta_{6}=0$, then Eq. (1.9) becomes a generalized Bogoyavlensky-Konopelchenko (BK) equation as cited in Refs. [34-36].

We address solving the fractional generalized CBS-BK equation in the sense of the modified Riemann-Liouville derivative which has been derived by [37]. These equations can be reduced to the nonlinear ordinary differential equations via integer orders utilizing some fractional complex transformations. Jumarie's modified Riemann-Liouville derivative of order $\alpha$ is given by

$$
D_{t}^{\alpha} u(t)= \begin{cases}\frac{1}{\Gamma(1-\alpha)} \int_{0}^{t}(t-\tau)^{-\alpha}(u(\tau)-u(0)) \mathrm{d} \tau & \text { if } 0<\alpha \leq 1 \\ {\left[u^{(n)}(t)\right]^{(\alpha-n)}} & \text { if } n \leq \alpha<n+1, n \geq 1 .\end{cases}
$$

We list some valuable properties of the Riemann-Liouville fractional derivative [38-40] as follows:

$$
\left\{\begin{aligned}
& D_{J}^{\alpha}(f(x) g(x))= \sum_{j=0}^{+\infty}\left(\begin{array}{c}
\alpha \\
j
\end{array}\right) f^{(j)}(x) g_{R-L}^{(\alpha-j)}(x)-\frac{f(0) g(0)}{x^{\alpha} \Gamma(1-\alpha)}, \\
& D_{J}^{\alpha}(f(g(x)))= \sum_{j=0}^{+\infty}\left(\begin{array}{c}
\alpha \\
j
\end{array}\right) \frac{x^{j-\alpha}(j)}{\Gamma(j-\alpha+1)} \sum_{m=1}^{j} f^{(m)}(g) \sum \prod_{k=1}^{j} \frac{1}{P_{k} !}\left(\frac{g^{(k)}}{k !}\right)^{P_{k}} \\
&+\frac{f(g(x))-f(g(0))}{x^{\alpha} \Gamma(1-\alpha)}, \\
& D_{t}^{\alpha} t^{\gamma}=\frac{\Gamma(\gamma+1)}{\Gamma(1+\alpha-\gamma)} t^{\gamma-\alpha}, \gamma>0,
\end{aligned}\right.
$$

where $\Gamma$ denotes the Gamma function.

In this paper, we will study the multiple rogue waves for determining the multiple soliton solutions. The multiple rogue waves method used by some of powerful authors for the various nonlinear equations, including constructing rogue waves with a controllable center in the nonlinear systems [41], a $(3+1)$-dimensional Hirota bilinear equation [42], the generalized $(3+1)$-dimensional KP equation [43], and the Boussinesq equation [44]. There are many new papers about this fields, such as lump solutions (constructing the lump-soliton and mixed lump strip solutions of $(3+1)$-dimensional soliton equation [45]; utilizing the linear superposition principle to discuss the $(3+1)$-dimensional Boiti-LeonManna-Pempinelli equation [46]; obtaining periodic solutions for many non-linear evolution equations in the integrable systems theory [47]), rogue wave solutions (utilizing the Hirota bilinear form of the extended $(3+1)$-dimensional JM equation to find 30 classes of rogue wave type solutions [48]; resonant multiple wave solutions to some integrable soliton equations [49]). Some important work related with recent development in fractional calculus and its applications can be pointed out referring to the valuable papers containing studies of general fractional derivatives: theory, methods and applications by Yang [50]; anomalous diffusion equations with the decay exponential kernel by the Laplace transform [51]; new fractal nonlinear Burgers' equation arising in the acoustic signals propagation by Yang and Machado [52]; time fractional nonlinear diffusion equation from diffusion process by fractional Lie group approach [53]; the generalized time fractional diffusion equation by symmetry analysis [54]; investigating a time fractional nonlinear heat conduction equation with applications in mathematics physics, integrable system, fluid mechanics and nonlinear areas, by means of applying the fractional symmetry group method [55]; and determining the time fractional extended $(2+1)$-dimensional Zakharov-Kuznetsov equation in quantum magneto-plasmas by using a group analysis approach [56]. In [57], an operator-based framework for the construction of analytical soliton solutions to frac- 
tional differential equations was showed to fractional differential equations were mapped from Caputo algebra to Riemann-Liouville algebra.

The rest of this paper is structured as follows. The Hirota bilinear scheme has been summarized in Sect. 2. In Sects. 3-5, the lump solution, lump-kink, and lump-soliton solution to the fractional generalized CBS-BK equation, respectively, have been given. In Sect. 6, the conclusions have been given.

\section{Hirota bilinear method}

Take the fractional generalized CBS-BK of the form

$$
\begin{aligned}
\mathbb{P}_{g \mathrm{CBS}-\mathrm{BK}}(\Upsilon):= & \Psi_{\tau}+\Psi_{x x y}+3 \Psi_{x} \Psi_{y}+\delta_{1} \Psi_{y}+\delta_{2} \Psi_{x y y}+\delta_{3} \Psi_{x}+\delta_{4}\left(3 \Psi_{x}^{2}+\Psi_{x x x}\right) \\
& +\delta_{5}\left(3 \Psi_{x y y}^{2}+\Psi_{y y y y}\right)+\delta_{6}\left(3 \Psi_{y} \Psi_{x y y}+\Psi_{y y y}\right)=0 .
\end{aligned}
$$

Assume the Hirota derivatives according to the functions $\phi(x), \varphi(x)$ can be presented as

$$
\prod_{i=1}^{3} D_{\lambda_{i}}^{\pi_{i}} \phi \cdot \varphi=\left.\prod_{i=1}^{3}\left(\frac{\partial}{\partial \lambda_{i}}-\frac{\partial}{\partial \mu_{i}}\right)^{o_{i}} \phi(\lambda) \varphi(\mu)\right|_{\mu=\lambda},
$$

where the vectors $\lambda=\left(\lambda_{1}, \lambda_{2}, \lambda_{3}\right), \mu=\left(\mu_{1}, \mu_{2}, \mu_{3}\right)$ and $o_{1}, o_{2}, o_{3}$ are optional nonnegative integers. It is clear that the fractional generalized CBS-BK equation above possesses the following Hirota bilinear:

$$
\begin{aligned}
& \mathbb{B}_{g \mathrm{CBS}-\mathrm{BK}}(f) \\
& :=\left(\delta_{4} D_{x}^{4}+D_{x}^{3} D_{y}+\delta_{3} D_{x}^{2}+D_{x} D_{\tau}+\delta_{2} D_{y}^{2}+\delta_{1} D_{x} D_{y}+\delta_{5} D_{y}^{4}+\delta_{6} D_{y}^{3} D_{x}\right) f . f \\
& =2\left[\delta_{4}\left(f_{x x x x}-4 f_{x} f_{x x x}+3 f_{x x}^{2}\right)+\left(f f_{x x x y}-f_{x} f_{x x x}-3 f_{x} f_{x x y}+3 f_{x x} f_{x y}\right)\right. \\
& +\delta_{3}\left(f_{x x}-f_{x}^{2}\right)+\left(f_{x \tau}-f_{x} f_{\tau}\right) \\
& +\delta_{2}\left(f f_{y y}-f_{y}^{2}\right)+\delta_{1}\left(f f_{x y}-f_{x} f_{y}\right)+\delta_{5}\left(f f_{y y y}-4 f_{y} f_{y y y}+3 f_{y y}^{2}\right) \\
& \left.+\delta_{6}\left(f_{y y y x}-f_{x} f_{y y y}-3 f_{y} f_{y y x}+3 f_{y y} f_{y x}\right)\right] \text {. }
\end{aligned}
$$

We utilize the following relationship between the functions $f(x, y, \tau)$ and $\Psi(x, y, \tau)$ :

$$
\Psi(x, y, \tau)=\Psi_{0}+2(\ln f(x, y, \tau))_{x}, \quad \Phi(x, y, \tau)=2(\ln f(x, y, \tau))_{x x} .
$$

According to the Bell polynomial theories of soliton equations [58], we can obtain the following relationship:

$$
\mathbb{P}_{g \mathrm{CBS}-\mathrm{BK}}(\Psi)=\left[\frac{\mathbb{B}_{g \mathrm{CBS}-\mathrm{BK}}(f)}{f}\right]_{x}
$$

Theorem $2.1 f$ solves $(2.4)$ if and only if $\Psi=2(\ln f)_{x}$ is demonstrated to be a solution to Eq. (2.1),

$$
\left(\delta_{4} D_{x}^{4}+D_{x}^{3} D_{y}+\delta_{3} D_{x}^{2}+D_{x} D_{\tau}+\delta_{2} D_{y}^{2}+\delta_{1} D_{x} D_{y}+\delta_{5} D_{y}^{4}+\delta_{6} D_{y}^{3} D_{x}\right) f . f
$$




$$
\begin{aligned}
= & 2\left[\delta_{4}\left(f_{x x x x}-4 f_{x} f_{x x x}+3 f_{x x}^{2}\right)+\left(f f_{x x x y}-f_{y} f_{x x x}-3 f_{x} f_{x x y}+3 f_{x x} f_{x y}\right)\right. \\
& +\delta_{3}\left(f_{x x}-f_{x}^{2}\right)+\left(f_{x \tau}-f_{x} f_{\tau}\right) \\
& +\delta_{2}\left(f f_{y y}-f_{y}^{2}\right)+\delta_{1}\left(f f_{x y}-f_{x} f_{y}\right)+\delta_{5}\left(\left(f_{y y y y}-4 f_{y} f_{y y y}+3 f_{y y}^{2}\right)\right. \\
& \left.+\delta_{6}\left(f f_{y y y x}-f_{x x} f_{y y y}-3 f_{y} f_{y y x}+3 f_{y x} f_{y x}\right)\right] .
\end{aligned}
$$

Proof By supposing $\theta=\partial_{x}(\ln f)$, from Eq. (2.4), we get

$$
\Psi=2 \theta \quad \Longleftrightarrow \quad f=\exp \left(\frac{1}{2} \int \Psi \mathrm{d} x\right),
$$

then, by considering $f=\exp \left(\partial_{x}^{-1} \theta\right)$ and $f>0$, the expressions $f_{x}, f_{y}, f_{\tau}, f_{x x}, f_{y y}, f_{x y}, f_{x \tau}, f_{x x y}$, $f_{x y y}, f_{x x x}, f_{x x y y}$, and $f_{x x x x}$, respectively, can be written as

$$
\begin{aligned}
f_{x}= & \theta \exp \left(\partial_{x}^{-1} \theta\right), \\
f_{y}= & \partial_{x}^{-1} \theta_{y} \exp \left(\partial_{x}^{-1} \theta\right), \\
f_{\tau}= & \partial_{x}^{-1} \theta_{\tau} \exp \left(\partial_{x}^{-1} \theta\right), \\
f_{x x}= & \left(\theta^{2}+\theta_{x}\right) \exp \left(\partial_{x}^{-1} \theta\right), \\
f_{y y}= & \left(\left(\partial_{x}^{-1} \theta_{y}\right)^{2}+\partial_{x}^{-1} \theta_{y y}\right) \exp \left(\partial_{x}^{-1} \theta\right), \\
f_{x y}= & \left(\theta \partial_{x}^{-1} \theta_{y}+\theta_{y}\right) \exp \left(\partial_{x}^{-1} \theta\right), \\
f_{x \tau}= & \left(\theta \partial_{x}^{-1} \theta_{t}+\theta_{t}\right) \exp \left(\partial_{x}^{-1} \theta\right), \\
f_{x x x}= & \left(\theta^{3}+3 \theta \theta_{x}+\theta_{x x}\right) \exp \left(\partial_{x}^{-1} \theta\right), \\
f_{x x y}= & {\left[\left(\theta^{2}+\theta_{x}\right) \partial_{x}^{-1} \theta_{y}+2 \theta \theta_{y}+\theta_{x y}\right] \exp \left(\partial_{x}^{-1} \theta\right), } \\
f_{x y y}= & {\left[\theta\left(\partial_{x}^{-1} \theta_{y}\right)^{2}+\theta \partial_{x}^{-1} \theta_{y y}+2 \theta_{y} \partial_{x}^{-1} \theta_{y}+\theta_{y y}\right] \exp \left(\partial_{x}^{-1} \theta\right), } \\
f_{x x x x}= & {\left[\theta^{4}+6 \theta^{2} \theta_{x}+4 \theta \theta_{x x}+3\left(\theta_{x}\right)^{2}+\theta_{x x x}\right] \exp \left(\partial_{x}^{-1} \theta\right), } \\
f_{x x y y}= & {\left[\left(\theta^{2}+\theta_{x}\right)\left(\left(\partial_{x}^{-1} \theta_{y}\right)^{2}+\partial_{x}^{-1} \theta_{y y}\right)+4 \theta \theta_{y} \partial_{x}^{-1} \theta_{y}+2 \theta_{x y} \partial_{x}^{-1} \theta_{y}\right.} \\
& \left.+2 \theta \theta_{y y}+2\left(\theta_{y}\right)^{2}+\theta_{x y y}\right] \exp \left(\partial_{x}^{-1} \theta\right) .
\end{aligned}
$$

Plugging (2.8)-(2.19) into (2.3) yields the bilinear form of Eq. (2.3) as

$$
\begin{aligned}
& 2\left[\delta_{4}\left(f f_{x x x x}-4 f_{x} f_{x x x}+3 f_{x x}^{2}\right)+\left(f f_{x x x y}-f_{y} f_{x x x}-3 f_{x x} f_{x x y}+3 f_{x x} f_{x y}\right)\right. \\
& \quad+\delta_{3}\left(f f_{x x}-f_{x}^{2}\right)+\left(f f_{x \tau}-f_{x} f_{\tau}\right) \\
& \quad+\delta_{2}\left(f f_{y y}-f_{y}^{2}\right)+\delta_{1}\left(f f_{x y}-f_{x} f_{y}\right)+\delta_{5}\left(f f_{y y y}-4 f_{y} f_{y y y}+3 f_{y y}^{2}\right) \\
& \left.\quad+\delta_{6}\left(f f_{y y y x}-f_{x x} f_{y y y}-3 f_{y} f_{y y x}+3 f_{y y} f_{y x}\right)\right] \\
& =2 \exp \left(2 \partial_{x}^{-1} \theta\right)\left[6\left(\int \frac{\partial^{2}}{\partial y^{2}} \theta(x, y, \tau) \mathrm{d} x\right)^{2} \delta_{5}\right. \\
& \quad+6 \int \frac{\partial^{2}}{\partial y^{2}} \theta(x, y, \tau) \mathrm{d} x\left(\frac{\partial}{\partial y} \theta(x, y, \tau)\right) \delta_{6}+6\left(\frac{\partial}{\partial x} \theta(x, y, \tau)\right)^{2} \delta_{4} \\
& \quad+\left(\frac{\partial^{3}}{\partial x^{3}} \theta(x, y, \tau)\right) \delta_{4}+\int \frac{\partial^{4}}{\partial y^{4}} \theta(x, y, \tau) \mathrm{d} x \delta_{5}
\end{aligned}
$$




$$
\begin{aligned}
& +\int \frac{\partial^{2}}{\partial y^{2}} \theta(x, y, \tau) \mathrm{d} x \delta_{2}+6\left(\frac{\partial}{\partial x} \theta(x, y, \tau)\right) \frac{\partial}{\partial y} \theta(x, y, \tau) \\
& +\left(\frac{\partial}{\partial x} \theta(x, y, \tau)\right) \delta_{3}+\left(\frac{\partial^{3}}{\partial y^{3}} \theta(x, y, \tau)\right) \delta_{6}+\left(\frac{\partial}{\partial y} \theta(x, y, \tau)\right) \delta_{1} \\
& \left.+\frac{\partial^{3}}{\partial x^{2} \partial y} \theta(x, y, \tau)+\frac{\partial}{\partial \tau} \theta(x, y, \tau)\right] \\
& =2 f^{2}\left[6 \delta_{5}\left(\partial_{x}^{-1} \theta_{y y}\right)^{2}+6 \delta_{6} \theta_{y}\left(\partial_{x}^{-1} \theta_{y y}\right)+6 \delta_{4} \theta_{x}^{2}+\delta_{4} \theta_{x x x}+\delta_{5} \partial_{x}^{-1} \theta_{y y y y}+\delta_{2} \partial_{x}^{-1} \theta_{y y}+6 \theta_{x} \theta_{y}\right. \\
& \left.+\delta_{3} \theta_{x}+\delta_{6} \theta_{y y y}+\delta_{1} \theta_{y}+\theta_{x x y}+\theta_{\tau}\right]
\end{aligned}
$$

or it can be rewritten as

$$
\begin{aligned}
\frac{1}{f^{2}} 2[ & \delta_{4}\left(f_{x x x x}-4 f_{x} f_{x x x}+3 f_{x x}^{2}\right)+\left(f f_{x x x y}-f_{y} f_{x x x}-3 f_{x} f_{x x y}+3 f_{x x} f_{x y}\right) \\
& +\delta_{3}\left(f_{x x}-f_{x}^{2}\right)+\left(f f_{x \tau}-f_{x} f_{\tau}\right) \\
& +\delta_{2}\left(f_{y y}-f_{y}^{2}\right)+\delta_{1}\left(f f_{x y}-f_{x} f_{y}\right)+\delta_{5}\left(f_{y y y y}-4 f_{y} f_{y y y}+3 f_{y y}^{2}\right) \\
& \left.+\delta_{6}\left(f f_{y y y x}-f_{x} f_{y y y}-3 f_{x} f_{y y x}+3 f_{y x} f_{y x}\right)\right] \\
= & \frac{\left(\delta_{4} D_{x}^{4}+D_{x}^{3} D_{y}+\delta_{3} D_{x}^{2}+D_{x} D_{\tau}+\delta_{2} D_{y}^{2}+\delta_{1} D_{x} D_{y}+\delta_{5} D_{y}^{4}+\delta_{6} D_{y}^{3} D_{x}\right) \mathfrak{f} \cdot f}{2 f^{2}},
\end{aligned}
$$

in which $\theta=\frac{1}{2} \Psi=\partial_{x}(\ln f)$ and $\partial_{x}^{-1}(\cdot)=\int(\cdot) \mathrm{d} x$. Therefore, Eq. (2.21) is the fractional generalized CBS-BK equation. Therefore, the theorem is complete.

\section{Rogue wave solutions of a ( $2+1)$-D fractional gCBS-BK equation}

For Eq. (2.1) with the obtained nonlinear PDE containing $f$, we get the combinations of positive functions, called a lump solution function:

$$
\begin{aligned}
& f(x, y, \tau)=\left(\sum_{i=1}^{4} a_{i} x_{i}\right)^{2}+\left(\sum_{i=5}^{8} a_{i} x_{i}\right)^{2}+a_{9}, \\
& \left(x_{1}, x_{2}, x_{3}, x_{4}\right)=\left(x_{5}, x_{6}, x_{7}, x_{8}\right)=(x, y, \tau, 1)
\end{aligned}
$$

and $\tau=\frac{t^{\alpha}}{\Gamma(\alpha+1)}$ where $a_{i}, i=1, \ldots, 9$ are the optional parameters which we are to find subsequently. Plugging (3.1) into Eq. (2.6), we get ten sets of nonlinear algebraic equations and then collecting the coefficients including $t, x, y$ and a constant, we obtain the following results for determining the solution function $\Psi=\Psi_{0}+2 \partial_{x}(\ln f)$.

Case I:

$$
\begin{aligned}
& \Psi_{1}(x, y, t) \\
& =\Psi_{0}+\frac{4\left(\Omega a_{2} x+a_{2} y-\frac{a_{2}\left(\Omega^{2} \delta_{3}+\Omega \delta_{1}+\delta_{2}\right) t^{\alpha}}{\Omega \Gamma(\alpha+1)}+a_{4}\right) \Omega a_{2}+4\left(a_{5} x+\frac{a_{5} y}{\Omega}-\frac{a_{5}\left(\Omega^{2} \delta_{3}+\Omega \delta_{1}+\delta_{2}\right) t^{\alpha}}{\Omega^{2} \Gamma(\alpha+1)}+a_{8}\right) a_{5}}{\left(\Omega a_{2} x+a_{2} y-\frac{a_{2}\left(\Omega^{2} \delta_{3}+\Omega \delta_{1}+\delta_{2}\right) t^{\alpha}}{\Omega \Gamma(\alpha+1)}+a_{4}\right)^{2}+\left(a_{5} x+\frac{a_{5} y}{\Omega}-\frac{a_{5}\left(\Omega^{2} \delta_{3}+\Omega \delta_{1}+\delta_{2}\right) t^{\alpha}}{\Omega^{2} \Gamma(\alpha+1)}+a_{8}\right)^{2}+a_{9}},
\end{aligned}
$$

where $\Omega$ solves $\delta_{4} \Omega^{4}+\Omega^{3}+\delta_{6} \Omega+\delta_{5}=0, a_{9}>0$ and $\Psi_{1}(x, y, t)$ is the lump solution.

Case II:

$$
a_{1}=a_{1}, \quad a_{2}=a_{2},
$$




$$
\begin{aligned}
a_{3}= & -\frac{\left(a_{1}^{2}+a_{5}^{2}\right)\left(a_{1} \delta_{3}+a_{2} \delta_{1}\right)+\delta_{2}\left(a_{1} a_{2}^{2}-a_{1} a_{6}^{2}+2 a_{2} a_{5} a_{6}\right)}{a_{1}^{2}+a_{5}^{2}}, \quad a_{4}=a_{4}, \\
a_{5}= & a_{5}, \quad a_{7}=a_{7}, \\
a_{7}= & -\frac{\left(a_{1}^{2}+a_{5}^{2}\right)\left(a_{5} \delta_{3}+a_{6} \delta_{1}\right)+\delta_{2}\left(2 a_{1} a_{2} a_{6}-a_{2}^{2} a_{5}+a_{5} a_{6}^{2}\right)}{a_{1}^{2}+a_{5}^{2}}, \quad a_{8}=a_{8}, \\
a_{9}= & -\left(3 \left(\left(a_{1}^{2}+a_{5}^{2}\right)^{2}\left(a_{1} a_{2}+a_{5} a_{6}\right)+\delta_{4}\left(a_{1}{ }^{2}+a_{5}{ }^{2}\right)^{3}+\delta_{5}\left(a_{2}{ }^{2}+a_{6}{ }^{2}\right)^{2}\left(a_{1}{ }^{2}+a_{5}{ }^{2}\right)\right.\right. \\
& \left.\left.+\delta_{6}\left(a_{2}{ }^{2}+a_{6}{ }^{2}\right)\left(a_{1}{ }^{2}+a_{5}{ }^{2}\right)\left(a_{1} a_{2}+a_{5} a_{6}\right)\right)\right) \\
& /\left(\delta_{2}\left(a_{1} a_{6}-a_{2} a_{5}\right)^{2}\right),
\end{aligned}
$$

then the function $f$ will be

$$
\begin{aligned}
& f_{2}(x, y, t)=\left(a_{1} x+a_{2} y+a_{3} \frac{t^{\alpha}}{\Gamma(\alpha+1)}+a_{4}\right)^{2}+\left(a_{5} x+a_{6} y+a_{7} \frac{t^{\alpha}}{\Gamma(\alpha+1)}+a_{8}\right)^{2}+a_{9}, \\
& \Psi_{2}(x, y, t) \\
& =\Psi_{0}+\frac{4 a_{1}\left(a_{1} x+a_{2} y+a_{3} \frac{t^{\alpha}}{\Gamma(\alpha+1)}+a_{4}\right)+4 a_{5}\left(a_{5} x+a_{6} y+a_{7} \frac{t^{\alpha}}{\Gamma(\alpha+1)}+a_{8}\right)}{f_{2}(x, y, t)} .
\end{aligned}
$$

Also, indeed we need to be ensured of the well-posedness, the positivity of $f_{2}(x, y, t)$ and rational analysis and localization of the function $\Psi_{2}$, respectively, which can be stated as

$$
\begin{aligned}
& \Delta=\left|\begin{array}{cc}
a_{1} & -a_{5} \\
a_{5} & a_{1}
\end{array}\right| \neq 0, \\
& \delta_{2}\left(\Delta^{2}\left(a_{1} a_{2}+a_{5} a_{6}\right)+\delta_{4} \Delta^{3}+\delta_{5} \Delta\left(a_{2}{ }^{2}+a_{6}{ }^{2}\right)^{2}+\delta_{6} \Delta\left(a_{2}{ }^{2}+a_{6}{ }^{2}\right)\left(a_{1} a_{2}+a_{5} a_{6}\right)\right)<0,
\end{aligned}
$$

and

$$
a_{1} a_{6}-a_{2} a_{5} \neq 0 \text {. }
$$

Moreover, by selecting the suitable values of parameters, the analytical treatment of periodic wave solution is presented in Figs. 1-3 including 3D plot, contour plot, density plot, and 2D plot when three spaces arise at spaces $y=-5, y=0$, and $y=5$. By taking the new value parameters such as $a_{1}=1, a_{2}=2, a_{4}=2, a_{5}=3, a_{6}=1.2, a_{8}=1, \delta_{1}=$ $1, \delta_{2}=-5, \delta_{3}=1.5, \delta_{4}=1.2, \delta_{5}=1.4, \delta_{6}=1.5, \Psi_{0}=0, t=2$, the corresponding the moving velocity and moving pass of the obtained lump in Case II are $v=29.05009176$ and $y=-1.030640668 x+1.086367345 x$. Also, a 2D plot of the lump-soliton by selecting the values of the different fractional order $\alpha$ is depicted in Fig. 4.

Remark 3.1 Because of using a simple computation, the lump has two critical points, but we investigate only one point $\left(x_{1}, y_{1}\right)=\left(\frac{1}{a_{1} a_{6}-a_{2} a_{5}}\left(\frac{t^{\alpha}\left(a_{2} a_{7}-a_{3} a_{6}\right)}{\Gamma(\alpha+1)}+a_{2} a_{8}-a_{4} a_{6}\right)+\frac{\sqrt{a_{9}\left(a_{1}{ }^{2}+a_{5}{ }^{2}\right)}}{a_{1}^{2}+a_{5}^{2}}\right.$, $\left.-\frac{1}{a_{1} a_{6}-a_{2} a_{5}}\left(\frac{t^{\alpha}\left(a_{1} a_{7}-a_{3} a_{5}\right)}{\Gamma(\alpha+1)}+a_{1} a_{8}-a_{4} a_{5}\right)\right)$. At the point $\left(x_{1}, y_{1}\right)$, the second-order derivative 

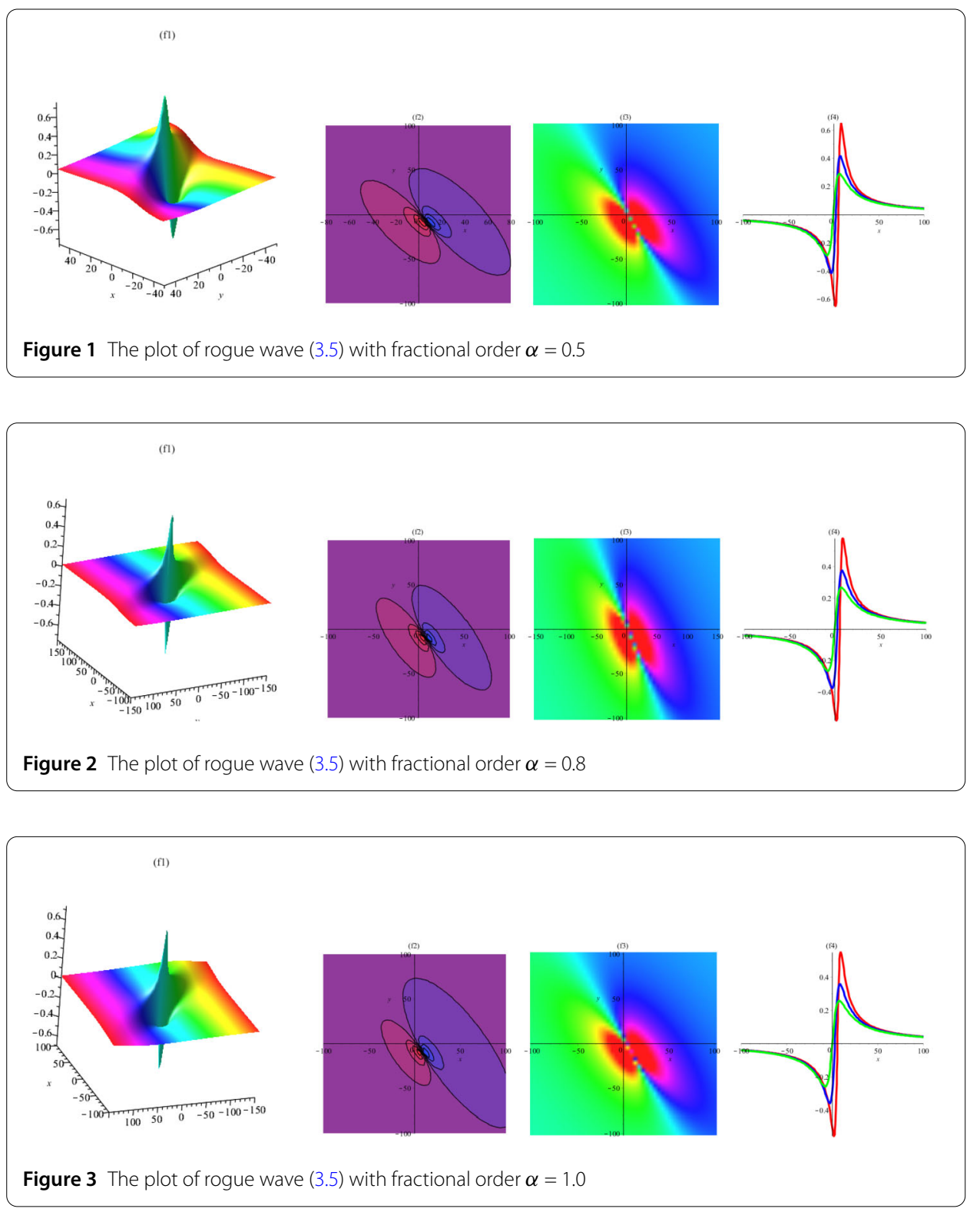

and the Hessian matrix can be determined given by [12]

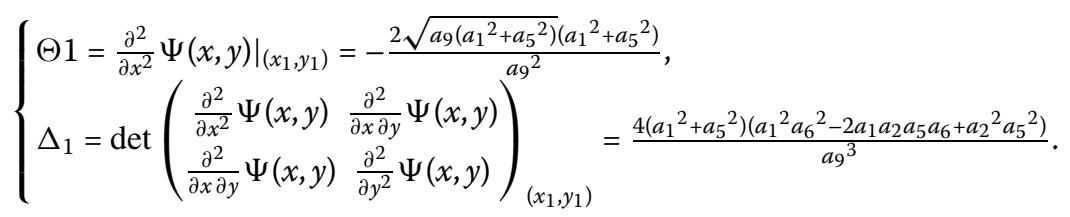

If $a_{9}{ }^{3}\left(a_{1}^{2} a_{6}{ }^{2}-2 a_{1} a_{2} a_{5} a_{6}+a_{2}^{2} a_{5}^{2}\right)>0$, then the point $\left(x_{1}, y_{1}\right)$ is an extreme value point. Based on above analysis, the point $\left(x_{1}, y_{1}\right)$ is a maximum value point at $\Psi_{\max }$. By using the different values of $\delta_{i}, i=1, \ldots, 5$, the lump solution $\Psi(x, y)$ has one maximum value containing

$$
\Psi_{\max }=\frac{2 \sqrt{a_{9}\left(a_{1}^{2}+a_{5}^{2}\right)}}{a_{9}} .
$$


Figure 4 The 2D plot of lump-soliton (3.5) with the different fractional orders

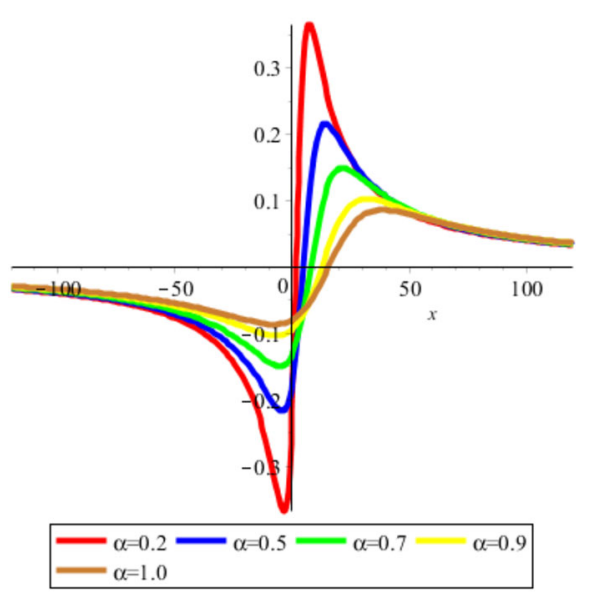

Remark 3.2 For three cases, it can be seen from Eqs. (3.3)-(3.5) in which the lump solution tends to 0 at any given time $t$ when $\left(\sum_{i=1}^{4} a_{i} x_{i}\right)^{2}+\left(\sum_{i=5}^{8} a_{i} x_{i}\right)^{2} \rightarrow 0$, or equivalently, $x^{2}+$ $y^{2} \rightarrow 0$. By utilizing

$$
\frac{\partial \Psi}{\partial x}=0, \quad \frac{\partial \Psi}{\partial y}=0
$$

in which $\Psi=\Psi_{0}+2 \partial_{x}\left(\ln \left(\sum_{i=1}^{4} a_{i} x_{i}\right)^{2}+\left(\sum_{i=5}^{8} a_{i} x_{i}\right)^{2}+a_{9}\right)$ the moving path of this lump can be determined as

$$
\left\{\begin{array}{l}
x=\frac{1}{a_{1} a_{6}-a_{2} a_{5}}\left(\frac{t^{\alpha}\left(a_{2} a_{7}-a_{3} a_{6}\right)}{\Gamma(\alpha+1)}+a_{2} a_{8}-a_{4} a_{6}\right)+\frac{\sqrt{a_{9}\left(a_{1}^{2}+a_{5}^{2}\right)}}{a_{1}^{2}+a_{5}^{2}} \\
y=-\frac{1}{a_{1} a_{6}-a_{2} a_{5}}\left(\frac{t^{\alpha}\left(a_{1} a_{7}-a_{3} a_{5}\right)}{\Gamma(\alpha+1)}+a_{1} a_{8}-a_{4} a_{5}\right)
\end{array}\right.
$$

also the moving velocity and the maximum amplitude, respectively, read

$$
v=\sqrt{\frac{\left(a_{2} a_{7}-a_{3} a_{6}\right)^{2}+\left(a_{1} a_{7}-a_{3} a_{5}\right)^{2}}{a_{1} a_{6}-a_{2} a_{5}}}, \quad H=4
$$

and the moving pass will be

$$
y=\frac{a_{3} a_{8}-a_{4} a_{7}}{a_{2} a_{7}-a_{3} a_{6}}+\frac{a_{1} a_{7}-a_{3} a_{5} \sqrt{a_{9}\left(a_{1}^{2}+a_{5}^{2}\right)}}{\left(a_{1}^{2}+a_{5}^{2}\right)\left(a_{2} a_{7}-a_{3} a_{6}\right)}-\frac{\left(a_{1} a_{7}-a_{3} a_{5}\right) x}{a_{2} a_{7}-a_{3} a_{6}} .
$$

It is worth mentioning that this rogue wave has the following features:

$$
\lim _{x \rightarrow \pm \infty} \Psi(x, y)=\Psi_{0}, \quad \lim _{y \rightarrow \pm \infty} \Psi(x, y)=\Psi_{0}
$$

\section{Lump-kink solutions}

In this section, for Eq. (2.1) with the obtained nonlinear PDE containing $f$, we get the combinations of positive functions and exponential function called a lump-kink solution. Necessary and sufficient conditions for positive quadratic functions to solve the Hirota 
bilinear equations conclude the study of lump solutions. Take the following function form:

$$
\begin{aligned}
& f(x, y, \tau)=\left(\sum_{i=1}^{4} a_{i} x_{i}\right)^{2}+\left(\sum_{i=5}^{8} a_{i} x_{i}\right)^{2}+\exp \left(\sum_{i=9}^{12} a_{i} x_{i}\right)+a_{13}, \\
& \left(x_{1,5,9}, x_{2,6,10}, x_{3,7,11}, x_{4,8,12}\right)=(x, y, \tau, 1)
\end{aligned}
$$

where $\tau=\frac{t^{\alpha}}{\Gamma(\alpha+1)}$ and $a_{i}, i=1, \ldots, 13$, are the optional parameters which we are to find subsequently. Putting (4.1) into Eq. (2.6), we get 20 sets of nonlinear algebraic equations and then collecting the coefficients including $e^{\sum_{i=9}^{12} a_{i} x_{i}}, \tau, x, y$ and a constant, we obtain the following results for finding the solution function $\Psi=\Psi_{0}+2 \partial_{x}(\ln f)$.

Case I:

$$
\begin{aligned}
& \Psi_{1}(x, y, t) \\
& =\Psi_{0}+\frac{4\left(a_{5} x+\frac{a_{7} t^{\alpha}}{\Gamma(\alpha+1)}+a_{8}\right) a_{5}}{\left(a_{2} y-\frac{a_{2} \delta_{1} t^{\alpha}}{\Gamma(\alpha+1)}+a_{4}\right)^{2}+\left(a_{5} x+\frac{a_{7} t^{\alpha}}{\Gamma(\alpha+1)}+a_{8}\right)^{2}+a_{13}+\mathrm{e}^{a_{10 y-} \frac{a_{10}\left(a_{10} \delta_{6}+\delta_{1}\right) t^{\alpha}}{\Gamma(\alpha+1)}+a_{12}}} .
\end{aligned}
$$

Case II:

$$
\begin{aligned}
& \Psi_{2}(x, y, t) \\
& \quad=\Psi_{0}+\frac{2 a_{9} \mathrm{e}^{-a_{9}\left(a_{9}{ }^{2} \delta_{4}+\delta_{3}\right) t+a_{9} x+a_{12}}}{\left(-t a_{2}\left(a_{9}{ }^{2}+\delta_{1}\right)+a_{2} y+a_{4}\right)^{2}+\left(\left(-a_{6} a_{9}{ }^{2}-a_{6} \delta_{1}\right) t+y a_{6}+a_{8}\right)^{2}+a_{13}+\mathrm{e}^{-a_{9}\left(a_{9}{ }^{2} \delta_{4}+\delta_{3}\right) t+a_{9} x+a_{12}}} .
\end{aligned}
$$

Case III:

$$
\begin{aligned}
f_{3}(x, y, t)= & \left(a_{2} y+\frac{a_{3} t^{\alpha}}{\Gamma(\alpha+1)}+a_{4}\right)^{2}+\left(a_{5} x+\frac{a_{5} a_{10} y}{a_{9}}+\frac{a_{7} t^{\alpha}}{\Gamma(\alpha+1)}+4 \frac{a_{5}}{a_{9}}\right)^{2} \\
& -\frac{1}{2} \frac{3 a_{2}^{2} a_{9}^{5}-2 a_{5}^{2} a_{10}{ }^{3} \delta_{2}}{a_{9}^{2} a_{10} \delta_{2}}+\mathrm{e}^{a_{9} x+a_{10} y-\frac{1}{3} \frac{a_{9}\left(2 a_{2}{ }_{2} \delta_{2}-3 a_{5} a_{7}\right) t^{\alpha}}{a_{5}{ }^{2} \Gamma(\alpha+1)}+a_{12}}
\end{aligned}
$$

$\Psi_{3}(x, y, t)$

$$
=\Psi_{0}+\frac{4\left(a_{5} x+\frac{a_{5} a_{10} y}{a_{9}}+\frac{a_{7} t^{\alpha}}{\Gamma(\alpha+1)}+4 \frac{a_{5}}{a_{9}}\right) a_{5}+2 a_{9} \mathrm{e}^{a_{9} x+a_{10} y-\frac{1}{3} \frac{a_{9}\left(2 a_{2}{ }^{2} \delta_{2}-3 a_{5} a_{7}\right) t^{\alpha}}{a_{5}{ }^{2} \Gamma(\alpha+1)}+a_{12}}}{f_{3}(x, y, t)} .
$$

Moreover, by selecting the suitable values of parameters, the analytical treatment of periodic wave solution is presented in Figs. 5 and 6 including 3D plot, contour plot, density plot, and 2D plot when three spaces arise at spaces $y=-5, y=0$, and $y=5$. By taking the new value parameters $a_{2}=1.2, a_{3}=1.4, a_{4}=2, a_{5}=1.5, a_{9}=1.7, a_{10}=1.2, a_{12}=1.5, \delta_{2}=$ $3, \Psi_{0}=1, t=2$, the obtained lump-kink solutions in Case II are presented with two different fractional orders. Also, 2D plot of the lump-soliton by selecting the values of the different fractional order $\alpha$ is depicted in Fig. 7.

Case IV:

$$
f_{4}(x, y, t)=\left(t a_{3}+\frac{\sqrt{a_{5}^{2} a_{10}^{2}-a_{6}^{2} a_{9}^{2}} y}{a_{9}}+a_{4}\right)^{2}+\left(a_{7} t+x a_{5}+y a_{6}+4 \frac{a_{5}}{a_{9}}\right)^{2}
$$




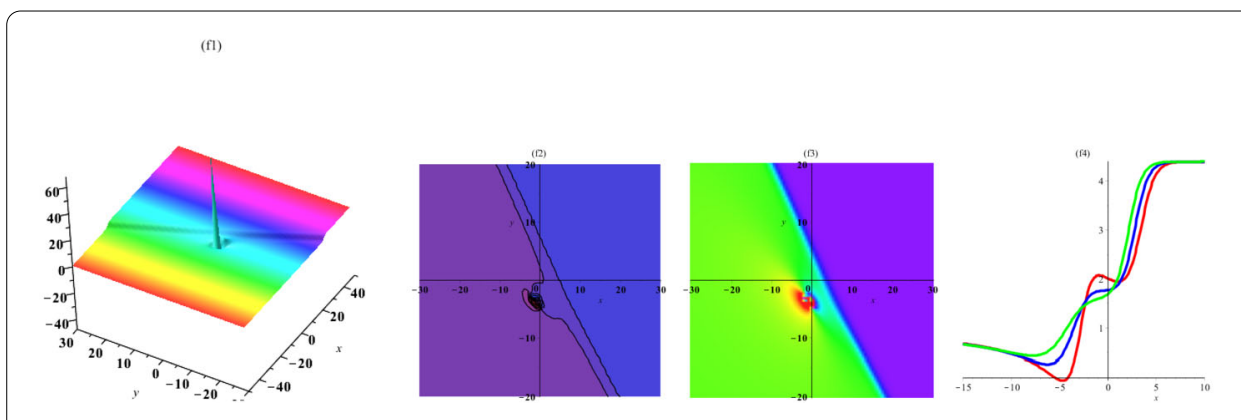

Figure 5 The plot of lump-kink (4.5) with fractional order $\alpha=0.5$

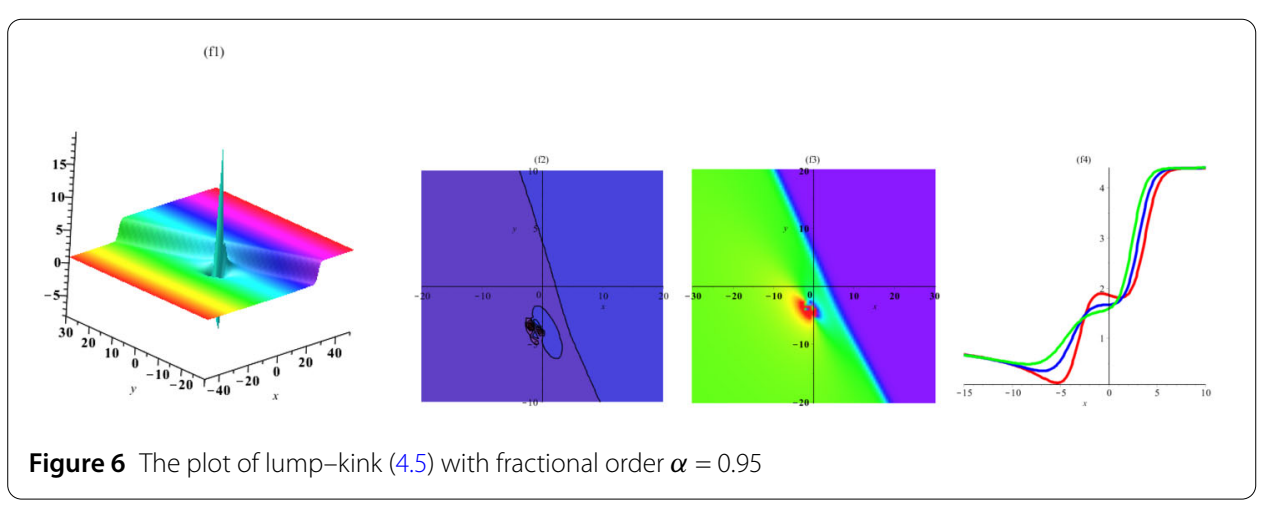

Figure 7 The 2D plot of lump-kink (4.5) with the different fractional orders

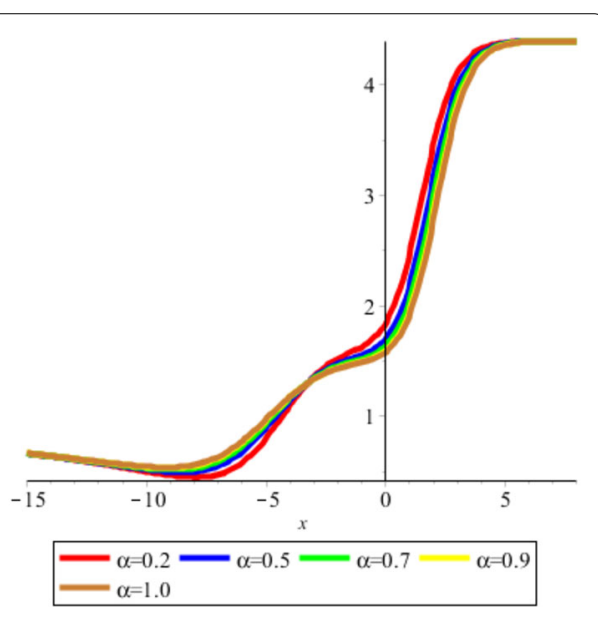

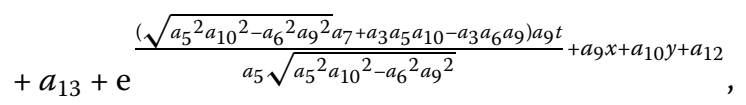

$\Psi_{4}(x, y, t)$

$$
=\Psi_{0}+\frac{4\left(a_{7} t+x a_{5}+y a_{6}+4 \frac{a_{5}}{a_{9}}\right) a_{5}+2 a_{9} \mathrm{e} \frac{\left(\sqrt{a_{5}^{2} a_{10}{ }^{2}-a_{6} a_{9}{ }^{2}} a_{7}+a_{3} a_{5} a_{10}-a_{3} a_{6} a_{9}\right) a_{9} t}{a_{5} \sqrt{a_{5}^{2} a_{10}{ }^{2}-a_{6} a_{9}^{2}}}+a_{9} x+a_{10} y+a_{12}}{f_{4}(x, y, t)} .
$$

\section{Case V:}

$\Psi_{4}(x, y, t)$ 


$$
=\Psi_{0}+\frac{4\left(-t a_{1} \delta_{3}+x a_{1}+a_{4}\right) a_{1}+2\left(-t a_{5} \delta_{3}+x a_{5}+a_{8}\right) a_{5}}{\left(-t a_{1} \delta_{3}+x a_{1}+a_{4}\right)^{2}+2\left(-t a_{5} \delta_{3}+x a_{5}+a_{8}\right)^{2}+a_{13}+\mathrm{e}^{\left(-a_{10} \delta_{6}-a_{10} \delta_{1}\right) t+a_{10} y+a_{12}}} .
$$

\section{Lump-soliton solutions}

In this section, to obtain to lump-soliton solutions, assume $f$ to be as follows:

$$
\begin{aligned}
& f(x, y, \tau)=\left(\sum_{i=1}^{4} a_{i} x_{i}\right)^{2}+\left(\sum_{i=5}^{8} a_{i} x_{i}\right)^{2}+\cosh \left(\sum_{i=9}^{12} a_{i} x_{i}\right)+a_{13} \\
& \left(x_{1,5,9}, x_{2,6,10}, x_{3,7,11}, x_{4,8,12}\right)=(x, y, \tau, 1)
\end{aligned}
$$

where $\tau=\frac{t^{\alpha}}{\Gamma(\alpha+1)}$ and $a_{i}, i=1, \ldots, 13$, are the optional parameters in which are to find subsequently. Substituting (4.8) into Eq. (2.6), we get 24 sets of nonlinear algebraic equations and then collecting the coefficients including $\cosh \left(\sum_{i=9}^{12} a_{i} x_{i}\right), \sinh \left(\sum_{i=9}^{12} a_{i} x_{i}\right), t, x, y$ and a constant, we obtain the following results for determining the solution function $\Psi=\Psi_{0}+2 \partial_{x}(\ln f)$.

Case I:

$$
\begin{aligned}
f_{1}= & \left(x a_{1}-\frac{y\left(a_{1}^{2} \delta_{4}+a_{5}^{2} \delta_{4}+a_{5} a_{6}\right)}{a_{1}}\right. \\
& \left.+\frac{\left(a_{1}^{2} \delta_{1} \delta_{4}+a_{5}^{2} \delta_{1} \delta_{4}-a_{1}^{2} \delta_{3}+a_{5} a_{6} \delta_{1}\right) t^{\alpha}}{a_{1} \Gamma(\alpha+1)}+a_{4}\right)^{2} \\
& +\left(x a_{5}+y a_{6}+\frac{\left(-a_{5} \delta_{3}-a_{6} \delta_{1}\right) t^{\alpha}}{\Gamma(\alpha+1)}+a_{8}\right)^{2}+a_{13}+\cosh \left(a_{10} y-\frac{a_{10} \delta_{1} t^{\alpha}}{\Gamma(\alpha+1)}+a_{12}\right), \\
\Psi_{1}= & \Psi_{0}+\left(4 \left(x a_{1}-\frac{y\left(a_{1}^{2} \delta_{4}+a_{5}^{2} \delta_{4}+a_{5} a_{6}\right)}{a_{1}}\right.\right. \\
& \left.+\frac{\left(a_{1}^{2} \delta_{1} \delta_{4}+a_{5}^{2} \delta_{1} \delta_{4}-a_{1}^{2} \delta_{3}+a_{5} a_{6} \delta_{1}\right) t^{\alpha}}{a_{1} \Gamma(\alpha+1)}+a_{4}\right) a_{1} \\
& \left.+4\left(x a_{5}+y a_{6}-\frac{\left(a_{5} \delta_{3}+a_{6} \delta_{1}\right) t^{\alpha}}{\Gamma(\alpha+1)}+a_{8}\right) a_{5}\right) / f_{1} .
\end{aligned}
$$

Case II:

$$
\begin{aligned}
f_{2}= & \left(x a_{1}-\frac{a_{5} a_{6} y}{a_{1}}-\frac{\left(a_{1}^{2} \delta_{3}+a_{5}^{2} \delta_{3}+a_{5} a_{7}\right) t^{\alpha}}{a_{1} \Gamma(\alpha+1)}+a_{4}\right)^{2} \\
& +\left(x a_{5}+y a_{6}+\frac{a_{7} t^{\alpha}}{\Gamma(\alpha+1)}+a_{8}\right)^{2}+a_{13} \\
& +\cosh \left(a_{10} y+\frac{a_{10}\left(-a_{6} a_{10}{ }^{2} \delta_{6}+a_{5} \delta_{3}+a_{7}\right) t^{\alpha}}{a_{6} \Gamma(\alpha+1)}+a_{12}\right), \\
\Psi_{2}= & \Psi_{0} \\
& +\frac{4\left(x a_{1}-\frac{a_{5} a_{6} y}{a_{1}}-\frac{\left(a_{1}^{2} \delta_{3}+a_{5}{ }^{2} \delta_{3}+a_{5} a_{7}\right) t^{\alpha}}{a_{1} \Gamma(\alpha+1)}+a_{4}\right) a_{1}+4\left(x a_{5}+y a_{6}+\frac{a_{7} t^{\alpha}}{\Gamma(\alpha+1)}+a_{8}\right) a_{5}}{f_{2}} .
\end{aligned}
$$


Case III:

$$
\begin{aligned}
& a_{1}=\frac{\sqrt{\left(16 \delta_{4} \delta_{5}{ }^{3}-\delta_{6}{ }^{4}-8 \delta_{5}{ }^{2} \delta_{6}\right)\left(-16 a_{5}{ }^{2} \delta_{4} \delta_{5}{ }^{3}+a_{5}{ }^{2} \delta_{6}{ }^{4}+8 a_{5}{ }^{2} \delta_{5}{ }^{2} \delta_{6}+2 \sqrt{-16 \delta_{2}{ }^{2} \delta_{4} \delta_{5}{ }^{5}+\delta_{2}{ }^{2} \delta_{5}{ }^{2} \delta_{6}{ }^{4}+8 \delta_{2}{ }^{2} \delta_{5}{ }^{4} \delta_{6}}\right)}}{16 \delta_{4} \delta_{5}{ }^{3}-\delta_{6}{ }^{4}-8 \delta_{5}{ }^{2} \delta_{6}}, \\
& a_{2}=\frac{1}{2} \frac{a_{1} a_{10}{ }^{2} \delta_{6}}{\delta_{2}}, \quad a_{3}=-\frac{1}{4} \frac{a_{1}\left(a_{10}{ }^{4} \delta_{6}{ }^{2}-2 a_{10} a_{11} \delta_{6}+4 \delta_{2} \delta_{3}\right)}{\delta_{2}}, \quad a_{4}=a_{4}, \\
& a_{5}=a_{5}, \quad a_{6}=\frac{1}{2} \frac{a_{5} a_{10}{ }^{2} \delta_{6}}{\delta_{2}}, \quad a_{8}=a_{8}, \\
& a_{7}=-\frac{a_{5}\left(a_{10}{ }^{4} \delta_{6}{ }^{2}-2 a_{10} a_{11} \delta_{6}+4 \delta_{2} \delta_{3}\right)}{4 \delta_{2}}, \quad a_{9}, \\
& a_{10}=\frac{\sqrt{-\delta_{5} \delta_{2}}}{\delta_{5}}, \quad a_{11}=-\frac{\delta_{1} \sqrt{-\delta_{5} \delta_{2}}}{\delta_{5}}, \quad a_{12} \\
& \Psi_{3}=\Psi_{0}+\frac{a_{12}}{\left(a_{1} x+a_{2} y+\frac{a_{3} t^{\alpha}}{\Gamma(\alpha+1)}+a_{4}\right)^{2}+\left(a_{5} x+a_{6} y+\frac{a_{7} t^{\alpha}}{\Gamma(\alpha+1)}+a_{8}\right)^{2}+\mathrm{e}^{a_{10} y+\frac{a_{11} t^{\alpha}}{\Gamma(\alpha+1)}+a_{12}}+a_{13}} .
\end{aligned}
$$

The existence condition of solution is of the form

$$
\begin{aligned}
& \left(16 \delta_{4} \delta_{5}{ }^{3}-\delta_{6}{ }^{4}-8 \delta_{5}{ }^{2} \delta_{6}\right)\left(-16 a_{5}{ }^{2} \delta_{4} \delta_{5}{ }^{3}+a_{5}{ }^{2} \delta_{6}{ }^{4}+8 a_{5}{ }^{2} \delta_{5}{ }^{2} \delta_{6}\right. \\
& \left.+2 \sqrt{-16 \delta_{2}{ }^{2} \delta_{4} \delta_{5}{ }^{5}+\delta_{2}{ }^{2}{\delta_{5}}^{2} \delta_{6}{ }^{4}+8 \delta_{2}{ }^{2} \delta_{5}{ }^{4} \delta_{6}}\right)>0
\end{aligned}
$$

and

$$
16 \delta_{4} \delta_{5}{ }^{3}-\delta_{6}{ }^{4}-8 \delta_{5}{ }^{2} \delta_{6}<0 .
$$

Case IV:

$$
\begin{aligned}
f_{4}= & \left(-\frac{1}{4} \frac{t a_{1}\left(a_{10}{ }^{4} \delta_{6}{ }^{2}+2 a_{10}{ }^{2} \delta_{1} \delta_{6}+4 \delta_{2} \delta_{3}\right)}{\delta_{2}}+a_{1} x+1 / 2 \frac{y a_{1} a_{10}{ }^{2} \delta_{6}}{\delta_{2}}+a_{4}\right)^{2} \\
& +\left(-\frac{1}{4} \frac{t a_{5}\left(a_{10}{ }^{4} \delta_{6}{ }^{2}+2 a_{10}{ }^{2} \delta_{1} \delta_{6}+4 \delta_{2} \delta_{3}\right)}{\delta_{2}}+a_{5} x+\frac{y a_{5} a_{10}{ }^{2} \delta_{6}}{2 \delta_{2}}+\frac{a_{4} a_{5}}{a_{1}}\right)^{2} \\
& +a_{13}+\cosh \left(-t a_{10} \delta_{1}+a_{10} y+a_{12}\right), \\
\Psi_{4}= & \Psi_{0}+\frac{1}{f_{4}}\left[4\left(-\frac{t a_{1}\left(a_{10} \delta_{6}{ }^{2}+2 a_{10}{ }^{2} \delta_{1} \delta_{6}+4 \delta_{2} \delta_{3}\right)}{4 \delta_{2}}+a_{1} x+\frac{y a_{1} a_{10}{ }^{2} \delta_{6}}{2 \delta_{2}}+a_{4}\right) a_{1}\right. \\
& \left.+4\left(-\frac{t a_{5}\left(a_{10}{ }^{4} \delta_{6}{ }^{2}+2 a_{10}{ }^{2} \delta_{1} \delta_{6}+4 \delta_{2} \delta_{3}\right)}{4 \delta_{2}}+a_{5} x+\frac{y a_{5} a_{10}{ }^{2} \delta_{6}}{2 \delta_{2}}+\frac{a_{4} a_{5}}{a_{1}}\right) a_{5}\right],
\end{aligned}
$$

in which

$$
\begin{aligned}
& a_{10}=\frac{\sqrt{-\delta_{5} \delta_{2}}}{\delta_{5}}, \\
& a_{1}=\frac{\sqrt{\left(16 \delta_{4} \delta_{5}{ }^{3}-\delta_{6}{ }^{4}-8 \delta_{5}{ }^{2} \delta_{6}\right)\left(-a_{5}{ }^{2}\left(16 \delta_{4} \delta_{5}{ }^{3}-\delta_{6}{ }^{4}-8 \delta_{5}{ }^{2} \delta_{6}\right)+2 \sqrt{-\delta_{2}{ }^{2} \delta_{5}{ }^{2}\left(16 \delta_{4} \delta_{5}{ }^{3}-\delta_{6}{ }^{4}-8 \delta_{5}{ }^{2} \delta_{6}\right)}\right)}}{16 \delta_{4} \delta_{5}{ }^{3}-\delta_{6}{ }^{4}-8 \delta_{5}{ }^{2} \delta_{6}} .
\end{aligned}
$$


Case V:

$$
\begin{aligned}
& a_{1}=a_{1}, \quad a_{2}=\frac{1}{2} \frac{a_{9} a_{10}\left(2 a_{1}^{2} a_{13}-a_{9}^{2}\right)}{a_{1}\left(-a_{9}^{2} a_{13}+2 a_{1}^{2}\right)}, \quad a_{4}=a_{4}, \quad a_{5}=0, \\
& a_{8}=a_{8}, \quad a_{9}=a_{9}, \quad a_{10}=a_{10}, \quad a_{12}=a_{12}, \\
& a_{3}=-\frac{1}{2 a_{9}^{2}\left(-a_{9}{ }^{2} a_{13}+2 a_{1}^{2}\right)^{2} a_{1}^{3}}\left[a_{1}^{2} a_{9}^{3} a_{10} \delta_{1}\left(2 a_{1}{ }^{2} a_{13}-a_{9}{ }^{2}\right)\left(-a_{9}{ }^{2} a_{13}+2 a_{1}{ }^{2}\right)\right. \\
& -a_{10}{ }^{2} \delta_{2}\left(-2 a_{1}{ }^{4} a_{9}{ }^{4} a_{13}{ }^{2}-8 a_{1}{ }^{6} a_{9}{ }^{2} a_{13}+4 a_{1}{ }^{2} a_{9}{ }^{6} a_{13}+8 a_{1}{ }^{8}-a_{9}{ }^{8}\right) \\
& \left.+2 a_{1}^{4} a_{9}^{2} \delta_{3}\left(-a_{9}^{2} a_{13}+2 a_{1}^{2}\right)^{2}\right] \text {, } \\
& a_{6}=\frac{1}{2} \frac{a_{10} \sqrt{-16 a_{1}^{6} a_{9}^{2} a_{13}+4 a_{1}^{2} a_{9}^{6} a_{13}+16 a_{1}^{8}-a_{9}^{8}}}{\left(-a_{9}^{2} a_{13}+2 a_{1}^{2}\right) a_{9} a_{1}}, \\
& a_{7}=-\frac{1}{2}\left(a _ { 1 0 } \sqrt { - 1 6 a _ { 1 } ^ { 6 } a _ { 9 } ^ { 2 } a _ { 1 3 } + 4 a _ { 1 } ^ { 2 } a _ { 9 } { } ^ { 6 } a _ { 1 3 } + 1 6 a _ { 1 } ^ { 8 } - a _ { 9 } ^ { 8 } } \left(a_{1}^{2} \delta_{1}\left(-a_{9}{ }^{2} a_{13}+2 a_{1}^{2}\right)\right.\right. \\
& \left.\left.+a_{9} a_{10} \delta_{2}\left(2 a_{1}^{2} a_{13}-a_{9}^{2}\right)\right)\right) \\
& /\left(\left(-a_{9}^{2} a_{13}+2 a_{1}^{2}\right)^{2} a_{9} a_{1}^{3}\right) \text {, } \\
& a_{11}=-\frac{1}{3} \frac{a_{10}{ }^{2} \delta_{2}\left(a_{9}{ }^{2} a_{13} a_{1}{ }^{2}+2 a_{1}^{4}-a_{9}^{4}\right)+3 a_{1}^{2} a_{9}\left(-a_{9}{ }^{2} a_{13}+2 a_{1}^{2}\right)\left(a_{9} \delta_{3}+a_{10} \delta_{1}\right)}{a_{1}^{2} a_{9}\left(-a_{9}{ }^{2} a_{13}+2 a_{1}{ }^{2}\right)}, \\
& f_{5}=\left(a_{1} x+a_{2} y+\frac{a_{3} t^{\alpha}}{\Gamma(\alpha+1)}+a_{4}\right)^{2}+\left(a_{6} y+\frac{a_{6} t^{\alpha}}{\Gamma(\alpha+1)}+a_{8}\right)^{2} \\
& +\cosh \left(a_{9} x+a_{10} y+\frac{a_{11} t^{\alpha}}{\Gamma(\alpha+1)}+a_{12}\right)+a_{13}, \\
& \Psi_{5}=\Psi_{0}+\frac{2 d f_{5} / d x}{f_{5}} .
\end{aligned}
$$

Also, by selecting the suitable values of parameters, the analytical treatment of periodic wave solution is presented in Figs. 8 and 9 including 3D plot, contour plot, density plot, and 2D plot when three spaces arise at spaces $x=-1, x=-3$, and $x=-5$. By taking the new value parameters $a_{1}=1.2, a_{4}=2, a_{5}=1.5, a_{8}=1.1, a_{9}=2, a_{10}=1, a_{12}=1.5, a_{13}=3, \delta_{1}=$ $2, \delta_{2}=-2, \delta_{3}=1.5, \delta_{6}=1.5, \Psi_{0}=1, y=1.5, t=2$, the obtained lump-soliton solutions in Case V are presented with two different fractional orders. Also, a 2D plot of the lumpsoliton by selecting values of the different fractional order $\alpha$ is depicted in Fig. 10. As pointed out above the positive polynomial functions and hyperbolic cosine function have the forms

$$
\begin{aligned}
& \Delta_{1}=\Delta_{11}^{2}=\left(a_{1} x+a_{2} y+\frac{a_{3} t^{\alpha}}{\Gamma(\alpha+1)}+a_{4}\right)^{2}, \quad \Delta_{2}=\Delta_{12}^{2}=\left(a_{6} y+\frac{a_{6} t^{\alpha}}{\Gamma(\alpha+1)}+a_{8}\right)^{2}, \\
& \Delta_{3}=\cosh \left(\Delta_{13}\right)=\cosh \left(a_{9} x+a_{10} y+\frac{a_{11} t^{\alpha}}{\Gamma(\alpha+1)}+a_{12}\right),
\end{aligned}
$$

by investigating the asymptotic behavior of a lump and resonance soliton pairs we have the following relations between $\Delta_{11}$ and $\Delta_{13}$ :

$$
\Delta_{11}=\frac{1}{2} \frac{a_{9}\left(2 a_{1}^{2} a_{13}-a_{9}^{2}\right)}{a_{1}\left(-a_{9}^{2} a_{13}+2 a_{1}^{2}\right)} \Delta_{13}+\frac{-4 a_{9}^{2} a_{13} a_{1}^{2}+4 a_{1}^{4}+a_{9}^{4}}{2 a_{1}\left(-a_{9}^{2} a_{13}+2 a_{1}^{2}\right)} x
$$




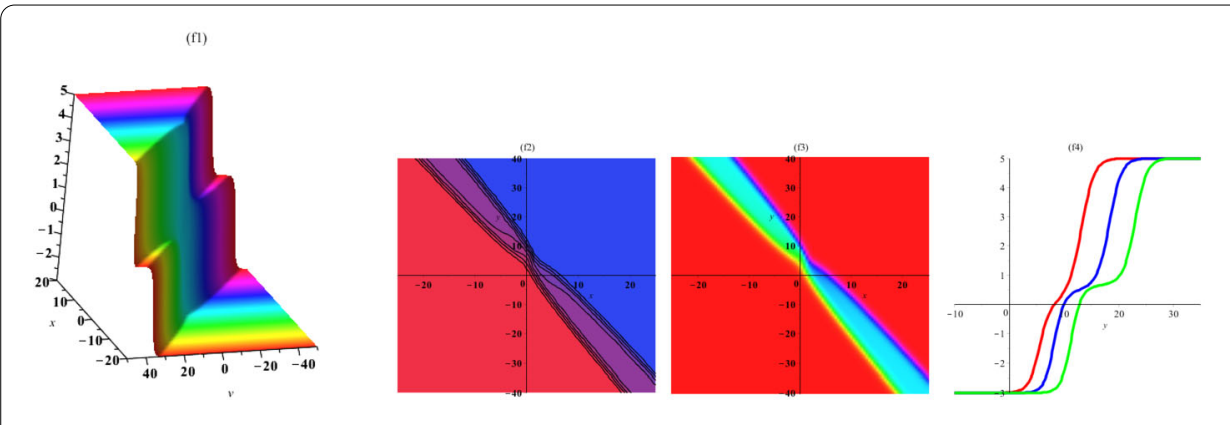

Figure 8 The plot of lump-soliton (5.11) with fractional order $\alpha=0.5$

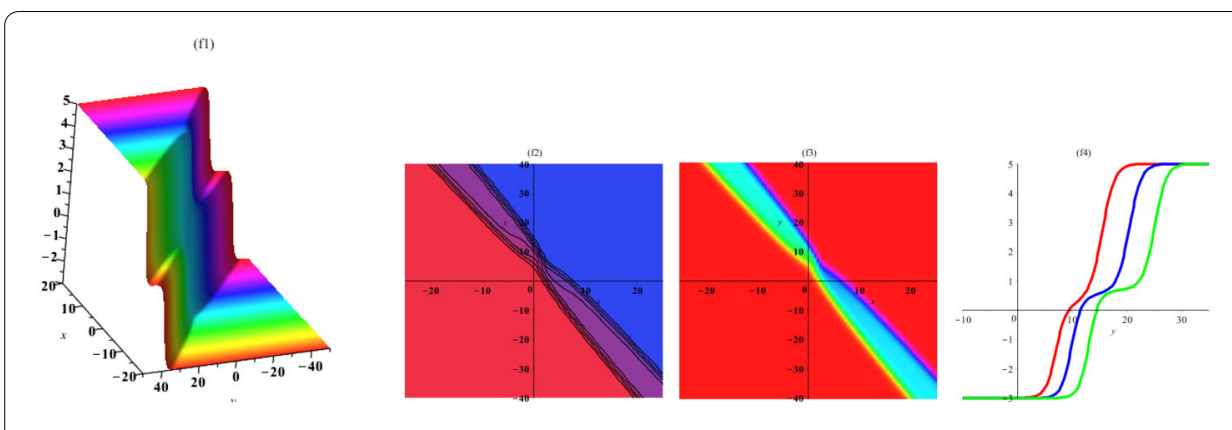

Figure 9 The plot of lump-soliton (5.11) with fractional order $\alpha=0.95$

$$
\begin{aligned}
& +\frac{-2 a_{1}^{2} a_{9} a_{12} a_{13}-2 a_{1} a_{4} a_{9}{ }^{2} a_{13}+4 a_{1}^{3} a_{4}+a_{9}{ }^{3} a_{12}}{2 a_{1}\left(-a_{9}{ }^{2} a_{13}+2 a_{1}^{2}\right)} \\
& -\frac{1}{6}\left(\left(-4 a_{9}{ }^{2} a_{13} a_{1}^{2}+4 a_{1}^{4}+a_{9}^{4}\right)\right. \\
& \left.\times\left(-3 a_{1}{ }^{2} a_{9}{ }^{4} a_{13} \delta_{3}-a_{1}{ }^{2} a_{9}{ }^{2} a_{10}{ }^{2} a_{13} \delta_{2}+6 a_{1}{ }^{4} a_{9}{ }^{2} \delta_{3}-6 a_{1}{ }^{4} a_{10}{ }^{2} \delta_{2}+2 a_{9}{ }^{4} a_{10}{ }^{2} \delta_{2}\right)\right) \\
& /\left(a_{9}{ }^{2}\left(-a_{9}{ }^{2} a_{13}+2 a_{1}{ }^{2}\right)^{2} a_{1}{ }^{3}\right) \frac{t^{\alpha}}{\Gamma(\alpha+1)},
\end{aligned}
$$

also the limited relations in which arise for $\Delta_{11}$ and $\Delta_{13}$ are of the form

$$
\begin{aligned}
\lim _{t \rightarrow \pm \infty} & \frac{\Delta_{11}}{\Delta_{12}} \\
= & \left(6 \Gamma(\alpha+1) a_{9}{ }^{2}\left(-a_{9}{ }^{2} a_{13}+2 a_{1}{ }^{2}\right)^{2} a_{1}{ }^{4}\right) \\
& /\left(\left(4 a_{1}^{4}+a_{9}^{4}-4 a_{9}{ }^{2} a_{13} a_{1}^{2}\right)\right. \\
& \left.\times\left(-3 a_{1}^{2} a_{9}^{4} a_{13} \delta_{3}-a_{1}{ }^{2} a_{9}{ }^{2} a_{10}{ }^{2} a_{13} \delta_{2}+6 a_{1}^{4} a_{9}{ }^{2} \delta_{3}-6 a_{1}{ }^{4} a_{10}{ }^{2} \delta_{2}+2 a_{9}{ }^{4} a_{10}{ }^{2} \delta_{2}\right)\right), \\
\lim _{t \rightarrow \pm \infty} & \frac{\Delta_{11}}{\Delta_{3}}=0, \quad \lim _{t \rightarrow \pm \infty} \frac{\Delta_{12}}{\Delta_{3}}=0,
\end{aligned}
$$

since one can consider $\Delta_{11}$ in its relation with $\Delta_{13}$, therefore at this time, the resonance soliton pairs occur when $\Delta_{11}$ contains $\Delta_{13}$.

Case VI:

$$
f_{6}=\left(\frac{t a_{1} a_{7}}{a_{5}}+x a_{1}+y a_{2}+a_{4}\right)^{2}+\left(t a_{7}+x a_{5}+\frac{y a_{2} a_{5}}{a_{1}}+a_{8}\right)^{2}+a_{13}
$$




$$
\begin{aligned}
& \text { Figure } 10 \text { The 2D plot of lump-soliton (5.11) with } \\
& \text { the different fractional orders } \\
& +\cosh \left(2 \frac{\left(2 a_{2}{ }^{3} a_{5} \delta_{6}\left(a_{1}{ }^{2}+a_{5}{ }^{2}\right)^{2}+a_{1}{ }^{2}\left(a_{1}{ }^{2}+a_{5}{ }^{2}\right)\left(2 a_{1}{ }^{2} a_{2} a_{5}+2 a_{2} a_{5}^{3}+2 a_{2} a_{5} \delta_{1}+a_{1} a_{7}\right)\right) t}{a_{1}{ }^{3} a_{5} \sqrt{2 a_{1}^{2}+2 a_{5}^{2}}}\right. \\
& \left.+x \sqrt{2 a_{1}^{2}+2 a_{5}^{2}}-\frac{y a_{2} \sqrt{2 a_{1}^{2}+2 a_{5}^{2}}}{a_{1}}+a_{12}\right), \quad \Psi_{6}=\Psi_{0}+\frac{d f_{6} / d x}{f_{6}}, \\
& \delta_{2}=-\frac{1}{2} \frac{-a_{2}^{2}\left(a_{1}^{2}+a_{5}^{2}\right)\left(3 a_{1} \delta_{6}-8 a_{2} \delta_{5}\right)+a_{1}^{5}+a_{1}^{3} a_{5}^{2}}{a_{1}^{2} a_{2}}, \quad \delta_{4}=-\frac{a_{2}{ }^{4} \delta_{5}}{a_{1}{ }^{4}}, \\
& \delta_{3}=\frac{1}{2}\left(-2 a_{1}^{3} a_{2} a_{5} \delta_{1}-a_{2}{ }^{3} a_{5}\left(a_{1}^{2}+a_{5}^{2}\right)\left(3 a_{1} \delta_{6}-8 a_{2} \delta_{5}\right)\right. \\
& \left.+a_{1}\left(-3 a_{2}^{3} a_{5}^{3} \delta_{6}+a_{1}^{4} a_{2} a_{5}+a_{1}^{2} a_{2} a_{5}^{3}-2 a_{1}^{3} a_{7}\right)\right) \\
& /\left(a_{1}^{4} a_{5}\right) \text {. }
\end{aligned}
$$

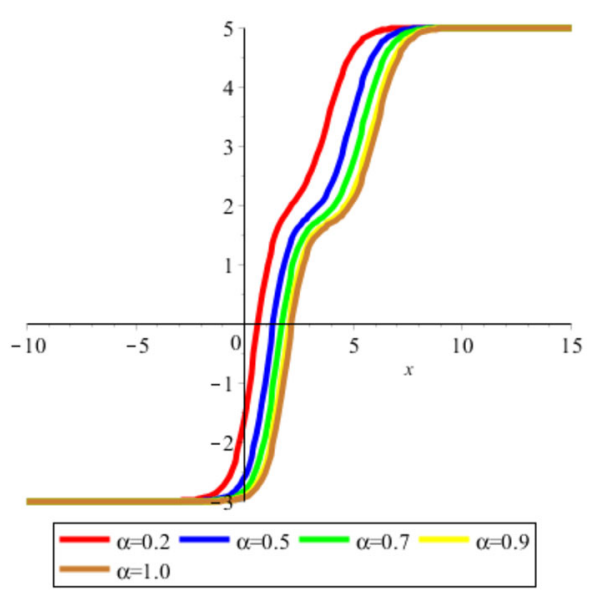

Remark In system (1.7), by utilizing $\alpha=1$ we get the original system. Also, by choosing the different $\alpha$ we can get attractive physical interpretations of the obtained solutions. In Figs. 1-10 the graphical illustrations of some solutions of the considered model have been plotted.

\section{Conclusion}

In this article, the localized waves, lump-solitons and solutions between lumps and rogue waves for the fractional generalized Calogero-Bogoyavlensky-Schiff-BogoyavlenskyKonopelchenko (CBS-BK) equation are investigated. The Hirota bilinear method is utilized which contain three cases including lump, lump-kink as the interaction between a lump and one line soliton and lump-soliton as the interaction between a lump and twoline solitons. The second-order derivative and the Hessian matrix for only one point investigated and the lump solution for the first-order rouge wave solution is obtained with one maximum value. The moving path of the lump solution and also the moving velocity and the maximum amplitude are obtained. The graphs for the various fractional order $\alpha$ are plotted containing 3D plot, contour plot, density plot and 2D plot. The results are beneficial to the study of the mathematics physics, fluid dynamics, and applied mechanics. All calculations in this paper have been made quickly with the aid of the Maple. 
Acknowledgements

Not applicable.

Funding

This work was not supported by any specific funding.

Availability of data and materials

The data sets supporting the conclusions of this article are included within the article and its additional file.

\section{Competing interests}

The authors declare that they have no competing interests.

\section{Authors' contributions}

The authors equally made contributions to this work. JM made the numerical simulations and wrote some sections of the article. OAI, LA, and AA provided the remaining sections. JM and AA provided the conclusions. Also, JM and OAl provided the references. The authors read and approved the final manuscript.

\section{Author details}

'Department of Applied Mathematics, Faculty of Mathematical Sciences, University of Tabriz, Tabriz, Iran. ${ }^{2}$ Department of Mathematics, Faculty of Education, Erciyes University, 38039, Melikgazi-Kayseri, Turkey. ${ }^{3}$ Division of Applied Mathematics, Brown University, 170 Hope St, Providence, RI 02906, USA. ${ }^{4}$ Department of Mechanical Engineering, College of Engineering, University of Zakho, Zakho, Iraq.

\section{Publisher's Note}

Springer Nature remains neutral with regard to jurisdictional claims in published maps and institutional affiliations.

Received: 10 June 2020 Accepted: 18 February 2021 Published online: 27 February 2021

\section{References}

1. Ali, K., Rizvi, S.T.R., Nawaz, B., Younis, M.: Optical solitons for paraxial wave equation in Kerr media. Mod. Phys. Lett. B 33(03), 1950020-1950029 (2019)

2. Arif, A., Younis, M., Imran, M., Tantawy, M., Rizvi, S.T.R.: Solitons and lump wave solutions to the graphene thermophoretic motion system with a variable heat transmission. Eur. Phys. J. Plus 134(6), 303 (2019)

3. Cattani, C., Sulaiman, T.A., Baskonus, H.M., Bulut, H.: Solitons in an inhomogeneous Murnaghan's rod. Eur. Phys. J. Plus $133,228(2018)$

4. Sulaiman, T.A., Bulut, H., Baskonus, H.M.: Investigation of the fractional coupled viscous Burgers' equation involving Mittag-Leffler kernel. Phys. A, Stat. Mech. Appl. 527, 121126 (2019)

5. Dehghan, M., Manafian, J., Saadatmandi, A.: Application of the Exp-function method for solving a partial differential equation arising in biology and population genetics. Int. J. Numer. Methods Heat Fluid Flow 21, 736-753 (2011)

6. Dehghan, M., Manafian, J., Saadatmandi, A.: Solving nonlinear fractional partial differential equations using the homotopy analysis method. Numer. Methods Partial Differ. Equ. 26, 448-479 (2010)

7. Dehghan, M., Manafian, J.: The solution of the variable coefficients fourth-order parabolic partial differential equations by homotopy perturbation method. Z. Naturforsch. A 64a, 420-430 (2009)

8. Sindi, C.T., Manafian, J.: Wave solutions for variants of the $K d V$-Burger and the $K(n, n)$-Burger equations by the generalized G'/G-expansion method. Math. Methods Appl. Sci. 40(12), 4350-4363 (2017)

9. Manafian, J., Lakestani, M.: Application of $\tan (\phi / 2)$-expansion method for solving the Biswas-Milovic equation for Kerr law nonlinearity. Optik 127, 2040-2054 (2016)

10. Seadawy, A.R., Manafian, J.: New soliton solution to the longitudinal wave equation in a magneto-electro-elastic circular rod. Results Phys. 8, 1158-1167 (2018)

11. Manafian, J.: Novel solitary wave solutions for the $(3+1)$-dimensional extended Jimbo-Miwa equations. Comput. Math. Appl. 76(5), 1246-1260 (2018)

12. Wang, C.J.: Spatiotemporal deformation of lump solution to $(2+1)$-dimensional KdV equation. Nonlinear Dyn. 84 697-702 (2016)

13. He, J.H.: A modified Li-He's variational principle for plasma. Int. J. Numer. Methods Heat Fluid Flow (2019). https://doi.org/10.1108/HFF-06-2019-0523

14. He, J.H.: Lagrange crisis and generalized variational principle for 3D unsteady flow. Int. J. Numer. Methods Heat Fluid Flow 30(3), 1189-1196 (2019)

15. Chen, S.S., Tian, B., Liu, L., Yuan, Y.Q., Zhang, C.R.: Conservation laws, binary Darboux transformations and solitons for a higher-order nonlinear Schrödinger system. Chaos Solitons Fractals 18, 337-346 (2019)

16. Du, X.X., Tian, B., Wu, X.Y., Yin, H.M., Zhang, C.R.: Lie group analysis, analytic solutions and conservation laws of the $(3+1)$-dimensional Zakharov-Kuznetsov-Burgers equation in a collisionless magnetized electron-positron-ion plasma. Eur. Phys. J. Plus 133, 378 (2018)

17. Saha Ray, S.: On conservation laws by Lie symmetry analysis for $(2+1)$-dimensional Bogoyavlensky-Konopelchenko equation in wave propagation. Comput. Math. Appl. 74, 1158-1165 (2017)

18. Zhao, X.H., Tian, B., Xie, X.Y., Wu, X.Y., Sun, Y., Guo, Y.J.: Solitons, Bäcklund transformation and Lax pair for a $(2+1)$-dimensional Davey-Stewartson system on surface waves of finite depth. Waves Random Complex Media 28, 356-366 (2018)

19. Abdullahi, R.A.: The generalized $(1+1)$-dimensional and $(2+1)$-dimensional Ito equations: multiple exp-function algorithm and multiple wave solutions. Comput. Math. Appl. 71, 1248-1258 (2016)

20. $\mathrm{Ma}, \mathrm{W} . \mathrm{X}$., Zhu, Z: Solving the $(3+1)$-dimensional generalized $\mathrm{kp}$ and bkp equations by the multiple exp-function algorithm. Appl. Math. Comput. 218(24), 11871-11879 (2012) 
21. Baskonus, H.M., Bulut, H.: Exponential prototype structures for $(2+1)$-dimensional Boiti-Leon-Pempinelli systems in mathematical physics. Waves Random Complex Media 26, 201-208 (2016)

22. Inc, M., Aliyu, A.I., Yusuf, A., Baleanu, D.: Optical solitary waves, conservation laws and modulation instabilty analysis to nonlinear Schrödinger's equations in compressional dispersive Alfvén waves. Optik 155, 257-266 (2018)

23. Bogoyavlenskii, O.I.: Breaking solitons in 2+ 1-dimensional integrable equations. Russ. Math. Surv. 45, 1-86 (1990)

24. Kudryasho, N., Pickering, A.: Rational solutions for Schwarzian integrable hierarchies. J. Phys. A 31, 9505-9518 (1998)

25. Clarkson, P.A., Gordoa, P.R., Pickering, A.: Multicomponent equations associated to non-isospectral scattering problems. Inverse Probl. 13, 1463-1476 (1997)

26. Estevez, P.G., Prada, J.: A generalization of the sine-Gordon equation (2+ 1)-dimensions. J. Nonlinear Math. Phys. 11, 168-179 (2004)

27. Zahran, E.H.M., Khater, M.M.A.: Modified extended tanh-function method and its applications to the Bogoyavlenskii equation. Appl. Math. Model. 40, 1769-1775 (2016)

28. Abadi, S.A.M., Naja, M.: Soliton solutions for $(2+1)$-dimensional breaking soliton equation: three wave method. Int. J. Appl. Math. Res. 1(2), 141-149 (2012)

29. Xin, X.P., Liu, X.Q., Zhang, L.L.: Explicit solutions of the Bogoyavlensky-Konoplechenko equation. Appl. Math. Comput. $215,3669-3673(2010)$

30. Prabhakar, M.V., Bhate, H.: Exact solutions of the Bogoyavlensky-Konoplechenko equation. Lett. Math. Phys. 64, 1-6 (2003)

31. Chen, S.T., Ma, W.X.: Exact solutions to a generalized Bogoyavlensky-Konopelchenko equation via maple symbolic computations. Complexity 2019, Article ID 8787460 (2019)

32. Hamid, M., Usman, M., Zubair, T., UI Haq, R., Shafee, A.: An efficient analysis for N-soliton, lump and lump-kink solutions of time-fractional (2 + 1)-Kadomtsev-Petviashvili equation. Phys. A, Stat. Mech. Appl. 528, 121320 (2019)

33. Ayub, K., Khan, M.Y., Mahmood-UI-Hassan, Q.: Solitary and periodic wave solutions of Calogero-Bogoyavlenskii-Schiff equation via exp-function methods. Comput. Math. Appl. 74, 3231-3241 (2017)

34. Chen, S.T., Ma, W.X.: Lump solutions of a generalized Calogero-Bogoyavlenskii-Schiff equation. Comput. Math. Appl. 76, 1680-1685 (2018)

35. Chen, S.T., Ma, W.X.: Lump solutions to a generalized Bogoyavlensky-Konopelchenko equation. Front. Math. China $13,525-534(2018)$

36. Bruzón, M.S., Gandarias, M.L., Muriel, C., Ramírez, J., Saez, S., Romero, F.R.: The Calogero-Bogoyavlenskii-Schiff equation in $2+1$ dimensions. Theor. Math. Phys. 137, 1367-1377 (2003)

37. Jumarie, G.: Modified Riemann-Liouville derivative and fractional Taylor series of nondifferentiable functions further results. Comput. Math. Appl. 51, 1367-1376 (2006)

38. Oldham, K.B., Spanier, J.: The Fractional Calculus. Academic Press, New York (1974)

39. Liu, C.S.: Counterexamples on Jumarie's two basic fractional calculus formulae. Commun. Nonlinear Sci. Numer. Simul. 22, 92-94 (2015)

40. Manafian, J., Lakestani, M.: A new analytical approach to solve some of the fractional-order partial differential equations. Indian J. Phys. 91(3), 243-258 (2017)

41. Zhaqilao: A symbolic computation approach to constructing rogue waves with a controllable center in the nonlinear systems. Comput. Math. Appl. 75(9), 3331-3342 (2018)

42. Liu, W., Zhang, Y.: Multiple rogue wave solutions for a $(3+1)$-dimensional Hirota bilinear equation. Appl. Math. Lett. 98, 184-190 (2019)

43. Zhang, H.Y., Zhang, Y.F.: Analysis on the M-rogue wave solutions of a generalized $(3+1)$-dimensional KP equation Appl. Math. Lett. 102, 106145 (2020)

44. Clarkson, P.A., Dowie, E.: Rational solutions of the Boussinesq equation and applications to rogue waves. Trans. Math. Appl. 1(1), 1-26 (2017)

45. Liu, J.G., Zhang, Y.: Construction of lump soliton and mixed lump stripe solutions of $(3+1)$-dimensional soliton equation. Results Phys. 10, 94-98 (2018)

46. Liu, J.G., Zhang, Y., Muhammad, I.: Resonant soliton and complexiton solutions for ( $3+1)$-dimensional Boiti-Leon-Manna-Pempinelli equation. Comput. Math. Appl. 75(11), 3939-3945 (2018)

47. Liu, J.G., Wu, P., Zhang, Y., Feng, L.: New periodic wave solutions of $(3+1)$-dimensional soliton equation. Therm. Sci. 21, 169-176 (2017)

48. Liu, J.G., Yang, X., Cheng, M., Feng, Y., Wang, Y.: Abound rogue wave type solutions to the extended (3 + 1)-dimensional Jimbo-Miwa equation. Comput. Math. Appl. 78, 1947-1959 (2019)

49. Liu, J.G., Yang, X.J., Feng, Y.Y., Wang, Y.: Resonant multiple wave solutions to some integrable soliton equations. Chin. Phys. B 28(11), 110202 (2019)

50. Yang, X.J.: General Fractional Derivatives: Theory, Methods and Applications. CRC Press, New York (2019)

51. Yang, X.J., Feng, Y.Y., Cattani, C., Inc, M.: Fundamental solutions of anomalous diffusion equations with the decay exponential kernel. Math. Methods Appl. Sci. 42, 4054-4060 (2019)

52. Yang, X.J., Tenreiro Machado, J.A.: A new fractal nonlinear Burgers' equation arising in the acoustic signals propagation. Math. Methods Appl. Sci. 42, 7539-7544 (2019)

53. Liu, J.G., Yang, X.J., Feng, Y.Y., Zhang, H.Y.: Analysis of the time fractional nonlinear diffusion equation from diffusion process. J. Appl. Anal. Comput. 10(3), 1060-1072 (2020)

54. Liu, J.G., Yang, X.J., Feng, Y.Y., Zhang, H.Y.: On the generalized time fractional diffusion equation: symmetry analysis, conservation laws, optimal system and exact solutions. Int. J. Geom. Methods Mod. Phys. 17(1), 2050013 (2020)

55. Liu, J.G., Yang, X.J., Feng, Y.Y.: On integrability of the time fractional nonlinear heat conduction equation. J. Geom. Phys. 144, 190-198 (2019)

56. Liu, J.G., Yang, X.J., Feng, Y.Y., Cui, P.: On group analysis of the time fractional extended (2+1)-dimensional Zakharov-Kuznetsov equation in quantum magneto-plasmas. Math. Comput. Simul. 178, 407-421 (2020)

57. Navickas, Z., Telksnys, T., Marcinkevicius, R., Ragulskis, M.: Operator-based approach for the construction of analytical soliton solutions to nonlinear fractional-order differential equations. Chaos Solitons Fractals 104, 625-634 (2017)

58. Liu, J.G., Yang, X.J., Feng, Y.Y.: On integrability of the extended (3 + 1)-dimensional Jimbo-Miwa equation. Math. Methods Appl. Sci. 43(4), 1646-1659 (2020) 
59. Ma, W.X., Zhou, Y.: Lump solutions to nonlinear partial differential equations via Hirota bilinear forms. J. Differ. Equ. 264, 2633-2659 (2018)

60. Ma, W.X.: A search for lump solutions to a combined fourth-order nonlinear PDE in (2+1)-dimensions. J. Appl. Anal. Comput. 9, 1319-1332 (2019)

61. Ma, W.X.: Interaction solutions to Hirota-Satsuma-Ito equation in (2+ 1)-dimensions. Front. Math. China 14, 619-629 (2019)

62. Ma, W.X.: Long-time asymptotics of a three-component coupled mKdV system. Mathematics 7(7), 573 (2019)

63. Manafian, J., Mohammadi-Ivatlo, B., Abapour, M.: Lump-type solutions and interaction phenomenon to the (2+1)-dimensional breaking soliton equation. Appl. Math. Comput. 13, 13-41 (2019)

64. Ilhan, O.A., Manafian, J., Shahriari, M.: Lump wave solutions and the interaction phenomenon for a variable-coefficient Kadomtsev-Petviashvili equation. Comput. Math. Appl. 78(8), 2429-2448 (2019)

65. IIhan, O.A., Manafian, J.: Periodic type and periodic cross-kink wave solutions to the $(2+1)$-dimensional breaking soliton equation arising in fluid dynamics. Mod. Phys. Lett. B 33, 1950277 (2019)

66. Ma, W.X., Zhou, Y., Dougherty, R.: Lump-type solutions to nonlinear differential equations derived from generalized bilinear equations. Int. J. Mod. Phys. B 30(28-29), 1640018 (2016)

67. Lü, J., Bilige, S., Gao, X., Bai, Y., Zhang, R.: Abundant lump solution and interaction phenomenon to Kadomtsev-Petviashvili-Benjamin-Bona-Mahony equation. J. Appl. Math. Phys. 6, 1733-1747 (2018)

68. Seyedi, S.H., Saray, B.N., Chamkha, A.J.: Heat and mass transfer investigation of MHD Eyring-Powell flow in a stretching channel with chemical reactions. Phys. A, Stat. Mech. Appl. 54415, 124109 (2020)

69. Sulaiman, T.A., Nuruddeen, R.I., Zerrad, E., Mikail, B.B.: Dark and singular solitons to the two nonlinear Schrödinger's equations. Optik 186, 423-430 (2019)

\section{Submit your manuscript to a SpringerOpen ${ }^{\circ}$ journal and benefit from:}

- Convenient online submission

- Rigorous peer review

- Open access: articles freely available online

- High visibility within the field

- Retaining the copyright to your article

Submit your next manuscript at $\gg$ springeropen.com 